\title{
Metric-affine approach to teleparallel gravity
}

\author{
Yu. N. Obukhov* and J. G. Pereira \\ Instituto de Física Teórica, Universidade Estadual Paulista, Rua Pamplona 145, 01405-900 São Paulo, Brazil
}

(Received 2 December 2002; published 27 February 2003)

\begin{abstract}
The teleparallel gravity theory, treated physically as a gauge theory of translations, naturally represents a particular case of the most general gauge-theoretic model based on the general affine group of spacetime. On the other hand, geometrically, the Weitzenböck spacetime of distant parallelism is a particular case of the general metric-affine spacetime manifold. These physical and geometrical facts offer a new approach to teleparallelism. We present a systematic treatment of teleparallel gravity within the framework of the metricaffine theory. The symmetries, conservation laws and the field equations are consistently derived, and the physical consequences are discussed in detail. We demonstrate that the so-called teleparallel GR-equivalent model has a number of attractive features which distinguishes it among the general teleparallel theories, although it has a consistency problem when dealing with spinning matter sources.
\end{abstract}

DOI: 10.1103/PhysRevD.67.044016

PACS number(s): 04.50.+h, 03.50.Kk, 04.20.Jb

\section{INTRODUCTION}

Theories of gravity based on the geometry of distant parallelism [1-7] are commonly considered as the closest alternative to general relativity (GR) theory. Teleparallel gravity models possess a number of attractive features both from the geometrical and physical viewpoints. Teleparallelism naturally arises within the framework of the gauge theory of the spacetime translation group. Translations are closely related to the group of general coordinate transformations which underlies GR. Accordingly, the energy-momentum current represents the matter source in the field equations of teleparallel gravity, as in GR.

Geometrically, teleparallel models are described by the Weitzenböck spacetime. The latter is characterized by a trivial curvature and nonzero torsion. The tetrad (or a coframe) field is the basic dynamical variable which can be treated as the gauge potential corresponding to the group of local translations. Then the torsion is naturally interpreted as the corresponding gauge field strength. As a result, a gravitational teleparallel Lagrangian is straightforwardly constructed, in a Yang-Mills manner, from the quadratic torsion invariants.

Teleparallel gravity belongs naturally to the class of the metric-affine gravitational theories (MAG). Quite generally [8], MAG can be understood within the framework of the gauge approach for the affine group, a semidirect product of the general linear group and the translation group. The corresponding gauge field potentials are the linear connection and the coframe, whereas the curvature and the torsion turn out to be the gauge gravitational field strengths. In addition, the metric represents one more fundamental field with the nonmetricity as the corresponding field strength.

From the gauge-theoretic point of view, teleparallel gravity is distinguished among the other MAG models by reducing the affine symmetry group to the translation subgroup. In geometric language, teleparallelism arises from the general

\footnotetext{
*On leave from Department of Theoretical Physics, Moscow State University, 117234 Moscow, Russia.
}

metric-affine spacetime after we impose two constraints by putting curvature and nonmetricity equal to zero. In this sense, teleparallelism can be treated, both physically and geometrically, as a particular case of the general MAG theory.

In the present paper we will study teleparallel gravity within the MAG approach. A similar analysis was previously developed in [6] for the Poincaré gauge approach. We will consider the general class of teleparallel models which are characterized by three dimensionless coupling constants. After recalling some basic facts about MAG in Sec. II, we derive the field equations of the general teleparallel theory in Sec. III. Then in Sec. IV we show that the teleparallel gravity model can be reduced to an effective Einstein theory with some modified energy-momentum current as a source. When the coupling constants have specific values (5.1), the exact equivalence between general relativity and teleparallel gravity is established for spinless sources. However, in Sec. V we demonstrate certain consistency problems for the sources with spin. Among the general teleparallel models, there is a class of theories with the proper conformal properties. We discuss the definition of conformal symmetry in teleparallelism in Sec. VI. Finally, we analyze the spherically symmetric solutions for general teleparallel gravity coupled to the electromagnetic field. We pay special attention to the behavior of the (Riemannian) curvature and torsion invariants. This aspect was not studied in detail before. The formulation of the spherically symmetric problem and its preliminary analysis is given in Secs. VII-IX. A number of new solutions is obtained, both charged and uncharged. In Sec. X we present the class of conformally flat solutions that generalizes the solutions of Bertotti and Robinson. The corresponding geometry appears to be regular from both the Riemannian curvature and the teleparallel torsion points of view. The complete set of uncharged solutions is presented in Sec. XI. This is done for all possible choices of the three coupling constants of teleparallel gravity. We analyze in Sec. XII the corresponding spacetime geometries, and find that the generic solutions are no black holes. In Sec. XIII we show that only in the above mentioned general relativity limit, i.e., for the specific values of the coupling constants (5.1), we recover the 
Schwarzschild and the Reissner-Nordström black hole configurations. This fact can be considered as an argument favoring the choice of the rigid structure of the teleparallel Lagrangian with the fixed values of the three coupling constants. The analysis of the torsion invariants reveals, however, that even for the case of the teleparallel equivalent of the general relativity, the horizon of a black hole represents a singular surface for the torsion. This latter fact was not noticed in the literature previously.

Our basic notations and conventions are those of [8]. In particular, the signature of the metric is assumed to be $(-$, $+,+,+)$. Spacetime coordinates are labeled by the Latin indices, $i, j, \ldots=0, \ldots, 3$ (for example, $d x^{i}$ ), whereas the Greek indices, $\alpha, \beta, \ldots=0, \ldots, 3$, label the local frame components (for example, the coframe 1-form $\vartheta^{\alpha}$ ). Along with the coframe 1 -forms $\vartheta^{\alpha}$, we will widely use the socalled $\eta$-basis of the dual coframes. Namely, we define the Hodge dual such that $\eta:=* 1$ is the volume 4-form. Furthermore, denoting by $e_{\alpha}$ the vector frame, we have that $\eta_{\alpha}$ $\left.\left.:=e_{\alpha}\right\rfloor \eta=* \vartheta_{\alpha}, \quad \eta_{\alpha \beta}:=e_{\beta}\right\rfloor \eta_{\alpha}=*\left(\vartheta_{\alpha} \wedge \vartheta_{\beta}\right), \quad \eta_{\alpha \beta \gamma}$ $\left.:=e_{\gamma}\right\rfloor \eta_{\alpha \beta}$, and $\left.\eta_{\alpha \beta \gamma \delta}:=e_{\delta}\right\rfloor \eta_{\alpha \beta \gamma}$. The last expression is thus the totally antisymmetric Levi-Civita tensor.

\section{PRELIMINARIES: METRIC-AFFINE APPROACH}

In this section we recall some basic facts concerning metric-affine geometry in four dimensions. For a more detailed discussion in arbitrary dimensions, see [8]. The metricaffine spacetime is described by the metric $g_{\alpha \beta}$, the coframe 1 -forms $\vartheta^{\alpha}$, and the linear connection 1 -forms $\Gamma_{\beta}{ }^{\alpha}$. These are interpreted as the generalized gauge potentials, while the corresponding field strengths are the nonmetricity 1-form $Q_{\alpha \beta}=-D g_{\alpha \beta}$, and the 2-forms of torsion $T^{\alpha}=D \vartheta^{\alpha}$ and curvature $R_{\beta}{ }^{\alpha}=d \Gamma_{\beta}{ }^{\alpha}+\Gamma_{\gamma}{ }^{\alpha} \wedge \Gamma_{\beta}{ }^{\gamma}$.

In our analysis of the teleparallel gravity we will heavily use the results related to the irreducible decompositions of the fundamental geometric and physical objects in MAG. The details about this method can be found in [8] and [9]. Here, we will mainly need to recall the irreducible parts of the torsion 2-form $T^{\alpha}$ which are defined by

$$
\begin{aligned}
& \left.{ }^{(2)} T^{\alpha}:=\frac{1}{3} \vartheta^{\alpha} \wedge T, \quad \text { with } \quad T:=e_{\alpha}\right\rfloor T^{\alpha}, \\
& { }^{(3)} T^{\alpha}:=-\frac{1}{3} *\left(\vartheta^{\alpha} \wedge P\right), \quad \text { with } P:=*\left(T^{\alpha} \wedge \vartheta_{\alpha}\right), \\
& { }^{(1)} T^{\alpha}:=T^{\alpha}-{ }^{(2)} T^{\alpha}-{ }^{(3)} T^{\alpha} .
\end{aligned}
$$

In tensor components, the torsion 2-form

$$
T^{\alpha}=\frac{1}{2} T_{i j}{ }^{\alpha} d x^{i} \wedge d x^{j}=\frac{1}{2} T_{\mu \nu}{ }^{\alpha} \vartheta^{\mu} \wedge \vartheta^{\nu}
$$

gives rise, through the above decomposition, to the vectors of trace and axial trace of torsion:

$$
\left.\left.e_{\alpha}\right\rfloor T=T_{\mu \alpha}^{\mu}, \quad e_{\alpha}\right\rfloor P=\frac{1}{2} T^{\mu \nu \lambda} \eta_{\mu \nu \lambda \alpha} .
$$

Given the metric $g_{\alpha \beta}$, the Christoffel connection $\widetilde{\Gamma}_{\beta}^{\alpha}$ is defined as a unique connection with vanishing torsion and nonmetricity: $\widetilde{D} \vartheta^{\alpha}=0$ and $\widetilde{D} g_{\alpha \beta}=0$. The Riemannian operators and geometrical objects, constructed from the Christoffel connection, will be denoted by a tilde. The general affine connection can always be decomposed into Riemannian and post-Riemannian parts,

$$
\Gamma_{\beta}^{\alpha}=\widetilde{\Gamma}_{\beta}^{\alpha}+N_{\beta}^{\alpha},
$$

where the distortion 1 -form $N_{\alpha \beta}$ can be expressed in terms of torsion and nonmetricity as

$$
\begin{aligned}
N_{\alpha \beta}= & \left.\left.\left.-e_{[\alpha} \mid T_{\beta]}+\frac{1}{2}\left(e_{\alpha}\right\rfloor e_{\beta}\right\rfloor T_{\gamma}\right) \vartheta^{\gamma}+\left(e_{[\alpha}\right\rfloor Q_{\beta] \gamma}\right) \vartheta^{\gamma} \\
& +\frac{1}{2} Q_{\alpha \beta} .
\end{aligned}
$$

The MAG Lagrangian of the gravitational field is constructed from the forms of curvature, torsion, and nonmetricity. In [9] the method of irreducible decompositions was applied to the most general quadratic Lagrangian, revealing the possibility of reformulating the MAG field equations as an effective Einstein equation. Here we will use the results of [9] to obtain an analogous reformulation of teleparallel gravity.

\section{FIELD EQUATIONS}

In the teleparallel theory, we have two geometrical constraints:

$$
\begin{gathered}
R_{\beta}{ }^{\alpha}=d \Gamma_{\beta}{ }^{\alpha}+\Gamma_{\gamma}{ }^{\alpha} \wedge \Gamma_{\beta}{ }^{\gamma}=0, \\
Q_{\alpha \beta}=-D g_{\alpha \beta}=-d g_{\alpha \beta}+\Gamma_{\beta}{ }^{\gamma} g_{\alpha \gamma}+\Gamma_{\alpha}{ }^{\gamma} g_{\alpha \gamma}=0 .
\end{gathered}
$$

These equations mean that there is a distant parallelism in spacetime. The result of a parallel transport of a vector does not depend on the path. Moreover, the lengths and angles are not changed during a parallel transport.

One may wonder, what actually we do gain when we "embed" teleparallel gravity into the MAG theory. After all, Eq. (3.1) may be solved trivially by performing a linear transformation of the frame and connection

$$
e_{\alpha} \rightarrow L_{\alpha}^{\beta} e_{\beta}, \quad \Gamma_{\alpha}^{\beta} \rightarrow L_{\alpha}^{\gamma} \Gamma_{\gamma}{ }^{\delta} L_{\delta}^{-1 \beta}+L_{\gamma}^{-1 \beta} d L_{\alpha}^{\gamma} .
$$

In view of Eqs. (3.1),(3.2), it is always possible to choose the matrix $L^{\beta}{ }_{\alpha}$ of the linear transformation in such a way that the transformed local metric becomes the standard Minkowski, $g_{\alpha \beta}=o_{\alpha \beta}:=\operatorname{diag}(-1,+1,+1,+1)$, whereas the transformed local connection becomes trivial: $\Gamma_{\alpha}{ }^{\beta}=0$. Then the (co)frame is left over as the only dynamical variable. We will call such a choice a Weitzenböck gauge. The visible 
disadvantage of such an approach is the resulting rigid structure of the tetrad field which cannot be altered at one's wish. To a great extent, this is contrary to the very spirit of relativity theory, which treats all coordinates and frame systems on an equal footing basis. By embedding teleparallelism into MAG, we remove the unwarranted rigidity of the tetrad field and return to the far more physically natural situation, where we can choose a reference and coordinate system at our best convenience.

Now we can formulate teleparallel gravity as the MAG model with the constraints (3.1),(3.2). Correspondingly, the Lagrangian of the teleparallel theory quite generally can be written as

$$
V=\frac{1}{2 \kappa} T^{\alpha} \wedge *\left(\sum_{I=1}^{3} a_{I}{ }^{(I)} T_{\alpha}\right)+\frac{1}{2} \mu^{\alpha \beta} \wedge Q_{\alpha \beta}-\nu^{\alpha}{ }_{\beta} \wedge R_{\alpha}{ }^{\beta} .
$$

Here $a_{1}, a_{2}, a_{3}$ are the three dimensionless coupling constants, and the gravitational coupling constant is, as usual, $\kappa=8 \pi G / c^{3}$, with $G$ the Newton's gravitational constant. Inserting Eqs. (2.1)-(2.4) into the first term in Eq. (3.4), we find

$$
\begin{aligned}
T^{\alpha} \wedge *\left(\sum_{I=1}^{3} a_{I}{ }^{(I)} T_{\alpha}\right)= & \left(c_{1} T_{\mu \nu}{ }^{\alpha} T^{\mu \nu}{ }_{\alpha}+c_{2} T_{\mu \alpha}{ }^{\mu} T^{\nu \alpha}{ }_{\nu}\right. \\
& \left.+c_{3} T_{\mu \nu}{ }^{\alpha} T_{\alpha}{ }^{\mu \nu}\right) \eta
\end{aligned}
$$

where the new coefficients are the following combinations of the original coupling constants:

$$
c_{1}=\frac{2 a_{1}+a_{3}}{3}, \quad c_{2}=\frac{2\left(a_{2}-a_{1}\right)}{3}, \quad c_{3}=\frac{2\left(a_{3}-a_{1}\right)}{3} .
$$

Variation of the teleparallel action with respect to the Lagrange multipliers $\mu^{\alpha \beta}$ and $\nu^{\alpha}{ }_{\beta}$ yields the constraints (3.1) and (3.2) of the geometry of distant parallelism. The Lagrangian (3.4) is invariant under the redefinition

$$
\begin{aligned}
& \mu^{\alpha \beta} \rightarrow \mu^{\alpha \beta}+D \xi^{\alpha \beta}, \\
& \nu_{\beta}^{\alpha} \rightarrow \nu_{\beta}^{\alpha}+D \chi^{\alpha}{ }_{\beta}-\xi^{\alpha}{ }_{\beta}
\end{aligned}
$$

of the Lagrange multiplier fields. Here, $\xi^{\alpha \beta}=\xi^{\beta \alpha}$ is an arbitrary symmetric 2 -form, whereas $\chi^{\alpha}{ }_{\beta}$ is an arbitrary 1-form. The proof of the invariance is based on the three Bianchi identities of MAG. Indeed, we have for the additional $\chi$ term arising from Eq. (3.8) in the Lagrangian

$$
\left(D \chi^{\alpha}{ }_{\beta}\right) \wedge R_{\alpha}{ }^{\beta}=d\left(\chi^{\alpha}{ }_{\beta} \wedge R_{\alpha}{ }^{\beta}\right)+\chi^{\alpha}{ }_{\beta} \wedge D R_{\alpha}{ }^{\beta} .
$$

Being a total differential, the first term in the right-hand side can be discarded. The last term vanishes in view of the Bianchi identity $D R_{\alpha}{ }^{\beta} \equiv 0$. As for the $\xi$ terms which appear in the Lagrangian when we make the transformation (3.7),(3.8), we find

$$
\begin{aligned}
\frac{1}{2}\left(D \xi^{\alpha \beta}\right) \wedge Q_{\alpha \beta}+\xi^{\alpha}{ }_{\beta} \wedge R_{\alpha}{ }^{\beta}= & \frac{1}{2} d\left(\xi^{\alpha \beta} \wedge Q_{\alpha \beta}\right)-\frac{1}{2} \xi^{\alpha \beta} \\
& \wedge\left(D Q_{\alpha \beta}-2 R_{(\alpha \beta)}\right) .
\end{aligned}
$$

Again, as a total derivative, the first term can be neglected, whereas the last term vanishes by virtue of the Bianchi identity $D Q_{\alpha \beta} \equiv 2 R_{(\alpha \beta)}$. As a result, the field equations derived from Eq. (3.4) will determine the Lagrange multipliers only up to the ambiguity imposed by the symmetry (3.7),(3.8).

The gravitational field equations are derived from the total Lagrangian $V+L_{\text {mat }}$ by independent variations with respect to the coframe $\vartheta^{\alpha}$ and connection $\Gamma_{\beta}{ }^{\alpha} 1$-forms. The corresponding so-called first and second field equations read

$$
\begin{gathered}
D H_{\alpha}-E_{\alpha}=\Sigma_{\alpha}, \\
D H^{\alpha}{ }_{\beta}-E^{\alpha}{ }_{\beta}=\Delta^{\alpha}{ }_{\beta} .
\end{gathered}
$$

The covector-valued forms appearing in the left-hand side of Eq. (3.11) are given by

$$
\begin{aligned}
H_{\alpha}:= & -\frac{\partial V}{\partial T^{\alpha}}=-\frac{1}{\kappa} *\left(\sum_{I=1}^{3} a_{I}{ }^{(I)} T_{\alpha}\right) \\
E_{\alpha}:= & \left.\left.e_{\alpha}\right\rfloor V+\left(e_{\alpha}\right\rfloor T^{\beta}\right) \wedge H_{\beta}=\frac{1}{2}\left[\left(e_{\alpha}\right\rfloor T^{\beta}\right) \wedge H_{\beta}-T^{\beta} \\
& \left.\left.\wedge\left(e_{\alpha}\right\rfloor H_{\beta}\right)\right] .
\end{aligned}
$$

In the last expression we have taken into account that $V=$ $-\frac{1}{2} T^{\alpha} \wedge H_{\alpha}$, in view of Eqs. (3.13) and (3.4).

The matter sources in the right-hand sides of (3.11) and (3.12) are the canonical energy-momentum current and the canonical spin current. For the minimally coupled matter field $\Psi^{A}$ ( $p$-form, in general), we have explicitly

$$
\begin{aligned}
\Sigma_{\alpha}:= & \left.\left.\left.\frac{\partial L_{\mathrm{mat}}}{\partial \vartheta^{\alpha}}=e_{\alpha}\right\rfloor L_{\mathrm{mat}}-\left(e_{\alpha}\right\rfloor D \Psi^{A}\right) \wedge \frac{\partial L_{\mathrm{mat}}}{\partial D \Psi^{A}}+\left(e_{\alpha}\right\rfloor \Psi^{A}\right) \\
& \wedge \frac{\partial L_{\mathrm{mat}}}{\partial \Psi^{A}} \\
\Delta_{\beta}^{\alpha}: & \frac{\partial L_{\mathrm{mat}}}{\partial \Gamma_{\alpha}{ }^{\beta}}=\left(\ell^{\alpha}{ }_{\beta}{ }_{B}{ }_{B} \Psi^{B}\right) \wedge \frac{\partial L_{\mathrm{mat}}}{\partial D \Psi^{A}}
\end{aligned}
$$

We will assume that the matter field $\Psi^{A}$ realizes some representation of the Lorentz group, and then $\ell_{\alpha \beta}=-\ell_{\beta \alpha}$ are the corresponding generators of infinitesimal Lorentz transformations. Accordingly, the hypermomentum current reduces to the spin current,

$$
\Delta_{\alpha \beta}=\tau_{\alpha \beta}=-\tau_{\beta \alpha} .
$$

The tensor-valued forms appearing in the left-hand side of the field equation (3.12) are given by 


$$
\begin{aligned}
& M^{\alpha \beta}:=-2 \frac{\partial V}{\partial Q_{\alpha \beta}}=\mu^{\alpha \beta}, \\
& H_{\beta}^{\alpha}:=-\frac{\partial V}{\partial R_{\alpha}^{\beta}}=\nu_{\beta}^{\alpha}, \\
& E_{\beta}^{\alpha}:=-\vartheta^{\alpha} \wedge H_{\beta}-M_{\beta}^{\alpha}=-\vartheta^{\alpha} \wedge H_{\beta}-\mu_{\beta}^{\alpha} .
\end{aligned}
$$

Substituting all this into Eq. (3.12), we find the equation

$$
\mu_{\beta}^{\alpha}+D \nu_{\beta}^{\alpha}=-\vartheta^{\alpha} \wedge H_{\beta}+\Delta_{\beta}^{\alpha} .
$$

The left-hand side of this equation is evidently invariant under the transformations (3.7),(3.8). Correspondingly, the field equation (3.21) offers maximum of the possible: It determines the gauge invariant piece of the Lagrange multipliers, namely $\mu_{\beta}^{\alpha}+D \nu_{\beta}^{\alpha}$, in terms of the spin current $\Delta_{\beta}^{\alpha}$ and of the translational field momentum $H_{\alpha}$.

It is important to notice that the Lagrange multipliers $\mu^{\alpha \beta}$ and $\nu_{\beta}^{\alpha}$ decouple from the first field equation (3.11). As a result, technically we can simply discard the second field equation (3.12) because it merely determines the Lagrange multipliers, whereas the dynamics of the gravitational field is governed by Eq. (3.11).

\section{EFFECTIVE EINSTEIN EQUATION}

In [9] it was demonstrated that a certain quadratic metricaffine Lagrangian has very special properties. This Lagrangian reads

$$
\begin{aligned}
V^{(0)}= & \frac{1}{2 \kappa}\left\{-R_{\alpha \beta} \wedge \eta^{\alpha \beta}-{ }^{(1)} T^{\alpha} \wedge *{ }^{(1)} T_{\alpha}+2^{(2)} T^{\alpha} \wedge{ }^{(2)} T_{\alpha}\right. \\
& +\frac{1}{2}{ }^{(3)} T^{\alpha} \wedge *{ }^{(3)} T_{\alpha}+{ }^{(2)} Q_{\alpha \beta} \wedge \vartheta^{\beta} \wedge *{ }^{(1)} T^{\alpha}-2^{(3)} Q_{\alpha \beta} \\
& \wedge \vartheta^{\beta} \wedge *{ }^{(2)} T^{\alpha}-2^{(4)} Q_{\alpha \beta} \wedge \vartheta^{\beta} \wedge *{ }^{(2)} T^{\alpha}+\frac{1}{4}{ }^{(1)} Q_{\alpha \beta} \\
& \wedge *{ }^{(1)} Q^{\alpha \beta}-\frac{1}{2}{ }^{(2)} Q_{\alpha \beta} \wedge *{ }^{(2)} Q^{\alpha \beta}-\frac{1}{8}{ }^{(3)} Q_{\alpha \beta} \\
& \wedge *{ }^{(3)} Q^{\alpha \beta}+\frac{3}{8}{ }^{(4)} Q_{\alpha \beta} \wedge *{ }^{(4)} Q^{\alpha \beta}+\left({ }^{(3)} Q_{\alpha \gamma} \wedge \vartheta^{\alpha}\right) \\
& \left.\wedge *\left({ }^{(4)} Q^{\beta \gamma} \wedge \vartheta_{\beta}\right)\right\} .
\end{aligned}
$$

The definition of the four irreducible parts of the nonmetricity ${ }^{(J)} Q_{\alpha \beta}, J=1, \ldots, 4$ is given in $[8,9]$; we do not need them here though because the linear and quadratic nonmetricity terms are zero anyway, in view of the teleparallel constraint (3.2).

The gravitational gauge field momenta for the Lagrangian (4.1) are given by

$$
\begin{aligned}
H_{\alpha}^{(0)} & :=-\frac{\partial V^{(0)}}{\partial T^{\alpha}} \equiv-\frac{1}{2 \kappa} N^{\mu \nu} \wedge \eta_{\alpha \mu \nu}, \quad H_{\beta}^{(0) \alpha}:=-\frac{\partial V^{(0)}}{\partial R_{\alpha}^{\beta}} \\
& =\frac{1}{2 \kappa} \eta_{\beta}^{\alpha},
\end{aligned}
$$

and the corresponding field equations coincide completely with Einstein's equations of general relativity:

$$
D H_{\alpha}^{(0)}-E_{\alpha}^{(0)} \equiv \frac{1}{2 \kappa} \widetilde{R}^{\mu \nu} \wedge \eta_{\alpha \mu \nu}, \quad D H_{\beta}^{(0) \alpha}-E_{\beta}^{(0) \alpha} \equiv 0 .
$$

We can use this fact and identically rewrite the teleparallel Lagrangian (3.4) as the sum

$$
V=-a_{1} V^{(0)}+\hat{V}
$$

where

$$
\begin{aligned}
\hat{V}= & \frac{1}{2 \kappa}\left(\alpha_{2} T^{\alpha} \wedge *{ }^{(2)} T_{\alpha}+\alpha_{3} T^{\alpha} \wedge *{ }^{(3)} T_{\alpha}\right)+\frac{1}{2} \mu^{\alpha \beta} \wedge Q_{\alpha \beta} \\
& -\nu^{\alpha}{ }_{\beta} \wedge R_{\alpha}{ }^{\beta},
\end{aligned}
$$

and the new "shifted" coupling constants are defined by

$$
\alpha_{2}=a_{2}+2 a_{1}, \quad \alpha_{3}=a_{3}+\frac{1}{2} a_{1}
$$

Correspondingly, the field momenta can be rewritten in the form

$$
\begin{aligned}
& M^{\alpha \beta}=-a_{1} M^{(0) \alpha \beta}+\hat{M}^{\alpha \beta}, \quad H_{\alpha}=-a_{1} H_{\alpha}^{(0)}+\hat{H}_{\alpha}, \\
& H_{\beta}^{\alpha}=-a_{1} H_{\beta}^{(0) \alpha}+\hat{H}_{\beta}^{\alpha}, \\
& E_{\alpha}=-a_{1} E_{\alpha}^{(0)}+\hat{E}_{\alpha}, \quad E_{\beta}^{\alpha}=-a_{1} E_{\beta}^{(0) \alpha}+\hat{E}_{\beta}^{\alpha} .
\end{aligned}
$$

We easily find that $\hat{M}^{\alpha \beta}:=-2 \partial \hat{V} / \partial Q_{\alpha \beta}=M^{\alpha \beta}$, and $\hat{H}_{\beta}^{\alpha}:=$ $-\partial \hat{V} / \partial R_{\alpha}^{\beta}=H_{\beta}^{\alpha}$, whereas

$$
\begin{aligned}
\hat{H}_{\alpha} & :=-\frac{\partial \hat{V}}{\partial T^{\alpha}}=-\frac{1}{\kappa} *\left(\alpha_{2}{ }^{(2)} T_{\alpha}+\alpha_{3}{ }^{(3)} T_{\alpha}\right) \\
& =-\frac{1}{3 \kappa}\left[\alpha_{2} *\left(\vartheta_{\alpha} \wedge T\right)+\alpha_{3} \vartheta_{\alpha} \wedge P\right]
\end{aligned}
$$

Here, we explicitly used the irreducible decompositions (2.1) and (2.2) of the torsion, in terms of the trace 1-form $T$ and the axial trace 1-form $P$. Then, with the help of the identities (4.3), one can transform the field equations (3.11) and (3.12) of MAG into

$$
\frac{-a_{1}}{2} \widetilde{R}^{\mu \nu} \wedge \eta_{\alpha \mu \nu}=\kappa\left(\Sigma_{\alpha}-D \hat{H}_{\alpha}+\hat{E}_{\alpha}\right)
$$




$$
\mu_{\beta}^{\alpha}+D \nu_{\beta}^{\alpha}=-\vartheta^{\alpha} \wedge \hat{H}_{\beta}+\Delta_{\beta}^{\alpha} .
$$

Accordingly, we can view the teleparallel field equations as the Einstein general relativity theory (4.10) with the effective energy-momentum current $\Sigma_{\alpha}^{\text {eff }}=\left(1 /-a_{1}\right)\left(\Sigma_{\alpha}-D \hat{H}_{\alpha}+\hat{E}_{\alpha}\right)$. Recall also that $\left.\left.\hat{E}_{\alpha}=e_{\alpha}\right\rfloor \hat{V}+\left(e_{\alpha}\right\rfloor T^{\beta}\right) \wedge \hat{H}_{\beta}$ and $D \hat{H}_{\alpha}=\widetilde{D} \hat{H}_{\alpha}$ $-N_{\alpha} \beta \wedge \hat{H}_{\beta}$. Now, substituting Eqs. (4.9) and (2.7), we find explicitly

$$
\begin{aligned}
D \hat{H}_{\alpha}-\hat{E}_{\alpha}= & \frac{\alpha_{2}}{3 \kappa}\left[-\eta_{\alpha \beta} \wedge \widetilde{D}\left(e^{\beta}\right] T\right)+* T_{\alpha} \wedge T-\frac{1}{2} \vartheta_{\alpha} \wedge P \wedge T \\
& \left.\left.-\frac{1}{2} e_{\alpha}\right\rfloor(T \wedge * T)\right]+\frac{\alpha_{3}}{3 \kappa}\left[\vartheta_{\alpha} \wedge d P-2 T_{\alpha} \wedge P+P\right. \\
& \left.\left.\left.\wedge e_{\alpha}\right\rfloor * P+\frac{1}{2} e_{\alpha}\right\rfloor(P \wedge * P)\right] .
\end{aligned}
$$

The effective Einstein equation (4.10) contains symmetric and antisymmetric parts. It is convenient to consider them separately. The antisymmetric piece is extracted by taking the interior product of $\left.e^{\alpha}\right\rfloor$ with Eq. (4.10). Taking into account Eq. (4.12), the result reads

$$
\begin{aligned}
& \left.2 \alpha_{3} d P-\alpha_{2}(* d T+P \wedge T)+e_{\alpha}\right\rfloor\left(\alpha_{2} * T^{\alpha} \wedge T-2 \alpha_{3} T^{\alpha} \wedge P\right) \\
& \left.\quad=3 \kappa e^{\alpha}\right\rfloor \Sigma_{\alpha} .
\end{aligned}
$$

We can now subtract the antisymmetric part from Eq. (4.10), which technically means substituting $d P$ from the above equation into the effective Einstein equation. As a result, we finally obtain

$$
\begin{aligned}
\frac{-a_{1}}{2} \widetilde{R}^{\mu \nu} \wedge \eta_{\alpha \mu \nu}= & \left.\kappa\left(\Sigma_{\alpha}-\frac{1}{2} \vartheta_{\alpha} \wedge e^{\beta}\right] \Sigma_{\beta}\right)+\frac{\alpha_{2}}{3}\left[\eta_{\alpha \beta}\right. \\
& \left.\wedge \widetilde{D}\left(e^{\beta}\right\rfloor T\right)-\frac{1}{2} \vartheta_{\alpha} \wedge * d T-* T_{\alpha} \wedge T \\
& \left.\left.\left.+\frac{1}{2} \vartheta_{\alpha} \wedge e_{\beta}\right\rfloor\left(* T^{\beta} \wedge T\right)+\frac{1}{2} e_{\alpha}\right\rfloor(T \wedge * T)\right] \\
& +\frac{\alpha_{3}}{3}\left[2 T_{\alpha} \wedge P-\vartheta_{\alpha} \wedge e_{\beta}\right\rfloor\left(T^{\beta} \wedge P\right)-P \\
& \left.\left.\wedge e_{\alpha}{ }^{*} P-\frac{1}{2} e_{\alpha}\right\rfloor(P \wedge * P)\right] .
\end{aligned}
$$

Analogously to the separation of the antisymmetric part, we can also extract the trace of the effective Einstein field equation. For this purpose, we multiply Eq. (4.14) with $\vartheta^{\alpha}$ $\wedge$ from the left, and we find

$$
\alpha_{2}\left(d^{*} T+T \wedge * T\right)+\frac{\alpha_{3}}{3} P \wedge * P=\kappa \vartheta^{\alpha} \wedge \Sigma_{\alpha}-a_{1} R \eta .
$$

Here, $R:=R_{\alpha \beta}{ }^{\beta \alpha}$ is the curvature scalar, and we used the identities: $\quad \vartheta^{\alpha} \wedge{ }^{*} T_{\alpha}=-{ }^{*} T, \quad \vartheta^{\alpha} \wedge \vartheta^{\beta} \wedge \eta_{\mu \nu}=2\left(\delta_{\mu}^{\alpha} \delta_{\nu}^{\beta}\right.$ $\left.-\delta_{\nu}^{\alpha} \delta_{\mu}^{\beta}\right) \eta$. Other useful formulas are $\left.e_{\alpha}\right\rfloor^{*} \psi=*\left(\psi \wedge \vartheta_{\alpha}\right)$ for any form $\psi$; also $\vartheta^{\alpha} \wedge \eta_{\beta}=\delta_{\beta}^{\alpha} \eta, \vartheta^{\alpha} \wedge \eta_{\mu \nu}=\delta_{\nu}^{\alpha} \eta_{\mu}-\delta_{\mu}^{\alpha} \eta_{\nu}$, and $\vartheta^{\alpha} \wedge \eta_{\mu \nu \lambda}=\delta_{\mu}^{\alpha} \eta_{\nu \lambda}+\delta_{\nu}^{\alpha} \eta_{\lambda \mu}+\delta_{\lambda}^{\alpha} \eta_{\mu \nu}$.

\section{GENERAL RELATIVITY LIMIT: A PROBLEM WITH SPINNING MATTER?}

When the coupling constants are chosen as

$$
a_{1}=-1, \quad a_{2}=2, \quad a_{3}=\frac{1}{2},
$$

we find from Eq. (4.6) that all $\alpha_{I}=0$. [In terms of the tensor reformulation (3.5), the relations (3.6) yield $c_{1}=-\frac{1}{2}, c_{2}$ $=2$, and $c_{3}=1$. These are the well-known values of the teleparallel equivalent of GR, as used in [3-5], e.g.] Consequently, $\hat{V}=0$, and thus $\hat{H}_{\alpha}=0$ and $\hat{E}_{\alpha}=0$. The teleparallel field equations (4.10) reduce to the general relativity theory, except for the fact that the physical source in the right-hand side of Eq. (4.10) is not the "metrical," but the canonical energy-momentum current $\Sigma_{\alpha}$. Thus one should be careful when the matter field has nontrivial spin.

In order to check the consistency of the teleparallel theory, we first notice that Eq. (4.11) forces the spin current to satisfy

$$
\tau_{\alpha \beta}=D \nu_{[\alpha \beta]} .
$$

Consequently, it must be conserved:

$$
D \tau_{\alpha \beta}=D D \nu_{[\alpha \beta]}=0 .
$$

As the next step, we multiply the Einstein equation (4.10) from the left by $\vartheta_{\beta}$. Then, making an antisymmetrization, we find that the antisymmetric piece of the energymomentum current must vanish:

$$
\vartheta_{[\beta} \wedge \Sigma_{\alpha]}=0 .
$$

This is a consequence of the symmetry of the Einstein tensor which is equivalent to the condition $\widetilde{R}^{\mu \nu} \wedge \vartheta_{[\beta} \wedge \eta_{\alpha] \mu \nu}=0$. The same conclusion is obtained directly from Eq. (4.13), in which $\alpha_{I}=0$ leads to the vanishing left-hand side. Recalling now the angular momentum conservation law,

$$
\vartheta_{[\beta} \wedge \Sigma_{\alpha]}+D \tau_{\beta \alpha}=0,
$$

we conclude that the spin current is separately conserved, in full agreement with Eq. (5.3).

Summarizing, the teleparallel gravity can consistently couple either to a spinless matter or to a matter with a conserved spin tensor. Since, for example, the Dirac spinor field does not have such properties, the teleparallel description of gravity is not applicable to that case.

\section{"New general relativity"}

The "new general relativity" $[1,2,12-14]$ model is defined by choosing the coupling constants as follows: 


$$
a_{1}=-1, \quad a_{2}=2, \quad a_{3} \neq \frac{1}{2}
$$

With this choice, the above inconsistency problem is avoided.

Indeed, now we have $\alpha_{3} \neq 0$, and hence Eq. (4.13) reduces to the meaningful equation for the axial trace of torsion:

$$
\left.\left.d P-e_{\alpha}\right\rfloor\left(T^{\alpha} \wedge P\right)=\frac{3 \kappa}{2 \alpha_{3}} e^{\alpha}\right\rfloor \Sigma_{\alpha}
$$

The right-hand side, which represents the antisymmetric part of the energy-momentum current, can be nontrivial now.

A different type of inconsistency which arises for this model was first noticed by Kopczyński [6] who has shown the existence of an "extra symmetry" of the Lagrangian which deforms the coframe (without touching the connection) in such a way that the axial trace remains invariant. Such a symmetry makes the theory physically nonpredictable because torsion is not determined uniquely by the field equations. Later, Nester [7] clarified that point by establishing conditions under which such a hidden symmetry can arise.

\section{CONFORMAL TRANSFORMATIONS IN TELEPARALLEL GRAVITY}

Conformal transformation in gravity is usually understood as the scaling of the line element

$$
d s^{2} \rightarrow \Omega^{2} d s^{2}, \quad \text { or equivalently, } \quad g_{i j} \rightarrow \Omega^{2} g_{i j} .
$$

The conformal factor $\Omega$ can be an arbitrary function of the spacetime coordinates. In teleparallel gravity, the above transformation is naturally realized as the scaling of the coframe:

$$
\vartheta^{\alpha} \rightarrow \Omega \vartheta^{\alpha}
$$

In terms of the components $\vartheta^{\alpha}=\vartheta_{i}^{\alpha} d x^{i}$, the conformal transformation reads

$$
\vartheta_{i}^{\alpha} \rightarrow \Omega \vartheta_{i}^{\alpha}, \text { and accordingly } g_{i j}=\vartheta_{i}^{\alpha} \vartheta_{j}^{\beta} g_{\alpha \beta} \rightarrow \Omega^{2} g_{i j}
$$

The local metric, which in the case of the orthonormal frames is equal to the Minkowski metric $g_{\alpha \beta}=o_{\alpha \beta}:=\operatorname{diag}$ $(-1,+1,+1,+1)$, is not affected by the scaling. The conformal transformation of the coframe (6.2) induces the scaling of the volume 4-form, $\eta:=\vartheta^{\hat{0}} \wedge \vartheta^{\hat{1}} \wedge \vartheta^{\hat{2}} \wedge \vartheta^{\hat{3}} \rightarrow \Omega^{4} \eta$. The dual frame vectors $e_{\alpha}$ evidently transform to $\Omega^{-1} e_{\alpha}$ under Eq. (6.2).

It is natural to assume that the local linear connection $\Gamma_{\beta}{ }^{\alpha}$ is conformally invariant. This is what we encounter in the realization of conformal symmetry in the Poincare gauge gravity [15-17]; see also the discussion in [18]. Then we conclude that the Weitzenböck conditions (3.1) and (3.2) are not changed by the conformal transformation. Consequently,
Eq. (6.2) leaves the fundamental structure of the teleparallel gravity untouched, and it describes a map between the different teleparallel models.

Unlike curvature and nonmetricity, the torsion 2-form (which plays the role of the gauge field strength in the teleparallel theory) transforms in a nontrivial way under the conformal scaling. Under the action of Eq. (6.2), we find

$$
T^{\alpha}=D \vartheta^{\alpha} \rightarrow \Omega T^{\alpha}+d \Omega \wedge \vartheta^{\alpha}
$$

As a result, the torsion trace 1-form changes as $T \rightarrow \Omega T$ $-3 d \Omega$, and hence only the second part of torsion (2.1) has the nontrivial transformation property

$$
{ }^{(2)} T^{\alpha} \rightarrow \Omega^{(2)} T^{\alpha}+d \Omega \wedge \vartheta^{\alpha},
$$

whereas the two other irreducible parts of torsion, given by Eqs. (2.2) and (2.3), transform covariantly:

$$
{ }^{(1)} T^{\alpha} \rightarrow \Omega^{(1)} T^{\alpha}, \quad{ }^{(3)} T^{\alpha} \rightarrow \Omega^{(3)} T^{\alpha} .
$$

Accordingly, the class of models (3.4), with a vanishing coupling constant $a_{2}=0$, will display the proper conformal behavior in the sense that the teleparallel Lagrangian is rescaled as

$$
V \rightarrow \Omega^{2} V
$$

Notice that, since the action is changed, it is therefore not conformal invariant.

We can use the general Lagrange-Noether machinery [8] developed for MAG to derive the corresponding properties of an arbitrary model with a proper conformal behavior. Let us consider a model with the most general Lagrangian $L$ $=L\left(\psi, D \psi, g_{\alpha \beta}, \vartheta^{\alpha}, \Gamma_{\alpha}^{\beta}, T^{\alpha}\right)$ for an arbitrary matter field $\psi$ interacting with the teleparallel gravitational field. Under the action of the above defined (infinitesimal) conformal transformation, the Lagrangian changes by

$$
\delta L=\delta \vartheta^{\alpha} \wedge \Sigma_{\alpha}+\delta \psi \wedge \frac{\partial L}{\partial \psi}+d\left[\delta \vartheta^{\alpha} \wedge \frac{\partial L}{\partial T^{\alpha}}+\delta \psi \wedge \frac{\partial L}{\partial D \psi}\right]
$$

Here, we took into account the conformal invariance of the metric and connection, $\delta g_{\alpha \beta}=0$ and $\delta \Gamma_{\alpha}{ }^{\beta}=0$. Suppose now that the matter field and the Lagrangian have the proper conformal behavior in the sense that, under the infinitesimal rescaling $(\Omega=1+\omega)$, we have

$$
\delta \psi=k \omega \psi, \quad \delta L=\ell \omega L,
$$

with the numbers $k$ and $\ell$ giving the conformal weight of the matter field and of the Lagrangian, respectively. Substituting this into Eq. (6.8), we find the two identities:

$$
\begin{gathered}
\vartheta^{\alpha} \wedge \frac{\partial L}{\partial T^{\alpha}}+k \psi \wedge \frac{\partial L}{\partial D \psi}=0, \\
\ell L-\vartheta^{\alpha} \wedge \Sigma_{\alpha}+\vartheta^{\alpha} \wedge D \frac{\partial L}{\partial T^{\alpha}}-T^{\alpha} \wedge \frac{\partial L}{\partial T^{\alpha}}
\end{gathered}
$$




$$
-D \psi \wedge \frac{\partial L}{\partial D \psi}-k \frac{\delta L}{\delta \psi}=0
$$

These arise, as usual [8], by considering the terms proportional to $\omega$ and $d \omega$ separately.

Specializing now to the case of the purely gravitational Lagrangian $L=V$, which does not depend on $\psi$ and has the conformal weight $\ell=2$ [see Eq. (6.7)], we obtain the two identities

$$
\vartheta^{\alpha} \wedge \frac{\partial V}{\partial T^{\alpha}}=0, \quad 2 V=\vartheta^{\alpha} \wedge \frac{\partial V}{\partial \vartheta^{\alpha}}+T^{\alpha} \wedge \frac{\partial V}{\partial T^{\alpha}}
$$

Turning to the teleparallel model (3.4) under consideration, we can verify explicitly that these identities are indeed valid for the case $a_{2}=0$ (note that $H_{\alpha}=-\partial V / \partial T^{\alpha}$ ).

Every extra invariance of the Lagrangian means that there is a certain arbitrariness in the classical solutions of the field equations. In particular, the above analysis shows that the solutions of the teleparallel models with $a_{2}=0$ can only be determined up to an arbitrary scale factor $\Omega$ of the coframe field. Correspondingly, in order to have a predictable teleparallel theory, we will mainly confine ourselves to the class of Lagrangians with $a_{2} \neq 0$.

\section{SPHERICAL SYMMETRY AND GEOMETRIC INVARIANTS}

Let us now proceed with the analysis of the classical solutions of the general teleparallel model. As a first step, we naturally turn our attention to the compact object configurations, and specifically to the case of spherical symmetry. It is worthwhile to note that such a study was never performed in full generality for the models with the three arbitrary coupling constants $a_{1}, a_{2}, a_{3}$. A partial analysis was done in [2].

As usual in the study of exact solutions, we have two complementary aspects. The first one concerns the convenient choice of the local coordinates and of the corresponding ansatz for the dynamical fields. The second aspect is to provide the invariant characterization of the resulting geometry. Roughly speaking, the choice of an ansatz helps to solve the field equations more easily, whereas the invariant description provides the correct understanding of the physical contents of a solution.

We look for a spherically symmetric solution with the line element

$$
g=-A^{2} d t^{2}+B^{2}\left(d r^{2}+r^{2} d \theta^{2}+r^{2} \sin ^{2} \theta d \phi^{2}\right) .
$$

The two functions $A=A(r)$ and $B=B(r)$ depend on the radial variable $r$. It is, however, not so trivial to come up with the ansatz for a tetrad. A convenient choice reads

$$
\vartheta^{\hat{0}}=A d t, \quad \vartheta^{\hat{1}}=B d r, \quad \vartheta^{\hat{2}}=B r d \theta, \quad \vartheta^{\hat{3}}=B r \sin \theta d \phi
$$

In addition, for the (non-Riemannian) connection we choose

$$
\Gamma_{2}^{1}=-\Gamma_{1}^{2}=-d \theta, \quad \Gamma_{3}^{1}=-\Gamma_{1}^{3}=-\sin \theta d \phi
$$

$$
\Gamma_{3}^{2}=-\Gamma_{2}^{3}=-\cos \theta d \phi
$$

It is easy to see that Eq. (7.3) is the pure gauge configuration, and the curvature is indeed vanishing, $R_{\beta}{ }^{\alpha}=d \Gamma_{\beta}{ }^{\alpha}+\Gamma_{\gamma}{ }^{\alpha}$ $\wedge \Gamma_{\beta}{ }^{\gamma}=0$. One can certainly perform a linear transformation which yields the Weitzenböck gauge, but the resulting tetrad ansatz becomes somewhat obscure, see [19], e.g. This point demonstrates the convenience of the metric-affine approach, which offers a greater flexibility in the choice of the ansatz for a solution.

Hereafter, derivatives with respect to the radial coordinate will be denoted by a prime. Although the curvature is zero, the torsion of the configuration (7.2),(7.3) is nontrivial and reads

$$
T^{\hat{0}}=-\frac{A^{\prime}}{A B} \vartheta^{\hat{0}} \wedge \vartheta^{\hat{1}}, \quad T^{\hat{2}}=\frac{B^{\prime}}{B^{2}} \vartheta^{\hat{1}} \wedge \vartheta^{\hat{2}}, \quad T^{\hat{3}}=\frac{B^{\prime}}{B^{2}} \vartheta^{\hat{1}} \wedge \vartheta^{\hat{3}}
$$

Correspondingly, the irreducible pieces of torsion are given by

$$
\begin{aligned}
{ }^{(1)} T^{\alpha}= & -\frac{\left(A^{\prime} B-B^{\prime} A\right)}{3 A B^{2}}\left(2 \delta_{\hat{0}}^{\alpha} \vartheta^{\hat{0}} \wedge \vartheta^{\hat{1}}+\delta_{\hat{2}}^{\alpha} \vartheta^{\hat{1}} \wedge \vartheta^{\hat{2}}+\delta_{\hat{3}}^{\alpha} \vartheta^{\hat{1}}\right. \\
& \left.\wedge \vartheta^{\hat{3}}\right)
\end{aligned}
$$

and

$$
\begin{aligned}
{ }^{(2)} T^{\alpha}= & \frac{\left(A^{\prime} B+2 B^{\prime} A\right)}{3 A B^{2}}\left(-\delta_{\hat{0}}^{\alpha} \vartheta^{\hat{0}} \wedge \vartheta^{\hat{1}}+\delta_{\hat{2}}^{\alpha} \vartheta^{\hat{1}} \wedge \vartheta^{\hat{2}}+\delta_{\hat{3}}^{\alpha} \vartheta^{\hat{1}}\right. \\
& \left.\wedge \vartheta^{\hat{3}}\right)
\end{aligned}
$$

The axial torsion vanishes identically: ${ }^{(3)} T^{\alpha}=0$.

The above ansatz is clearly a coordinate- and framedependent statement. In order to have a correct understanding of the resulting solution, we need to construct invariants of the curvature and torsion. The total (Riemann-Cartan) curvature is identically zero in the teleparallel gravity. However, the Riemannian curvature of the Christoffel connection for the metric (7.1) is nontrivial. In particular, computation of the Weyl 2-form yields

$$
\begin{gathered}
\widetilde{W}^{\hat{0} \hat{1}}=\frac{W}{3} \vartheta^{\hat{0}} \wedge \vartheta^{\hat{1}}, \quad \widetilde{W}^{\hat{0} \hat{2}}=-\frac{W}{6} \vartheta^{\hat{0}} \wedge \vartheta^{\hat{2}}, \\
\widetilde{W}^{\hat{0} \hat{3}}=-\frac{W}{6} \vartheta^{\hat{0}} \wedge \vartheta^{\hat{3}}, \\
\widetilde{W}^{\hat{1} \hat{2}}=-\frac{W}{6} \vartheta^{\hat{1}} \wedge \vartheta^{\hat{2}}, \quad \widetilde{W}^{\hat{3} \hat{1}}=-\frac{W}{6} \vartheta^{\hat{3}} \wedge \vartheta^{\hat{1}}, \\
\widetilde{W}^{\hat{2} \hat{3}}=\frac{W}{3} \vartheta^{\hat{2}} \wedge \vartheta^{\hat{3}},
\end{gathered}
$$

with 


$$
W=\frac{r}{A B}\left[\frac{1}{r}\left(\frac{A}{B}\right)^{\prime}\right]^{\prime}
$$

The components of this 2-form represent the Weyl tensor, $\widetilde{W}^{\alpha \beta}=\frac{1}{2} C_{\mu \nu}{ }^{\alpha \beta} \vartheta^{\mu} \wedge \vartheta^{\nu}$. The Weyl quadratic invariant thus reads

$$
\widetilde{W}_{\alpha \beta} \wedge * \widetilde{W}^{\alpha \beta}=\frac{W^{2}}{3} \eta
$$

and consequently we can consistently use Eq. (7.9) for the description of the resulting geometry.

Besides the Riemannian Weyl tensor, the spacetime geometry is naturally characterized by the quadratic invariants of the torsion. For the spherically symmetric configurations (7.4), we have explicitly

$$
T_{\alpha} \wedge * T^{\alpha}=\frac{1}{B^{2}}\left[\left(\frac{A^{\prime}}{A}\right)^{2}-2\left(\frac{B^{\prime}}{B}\right)^{2}\right] \eta
$$

These two invariants-the Riemannian curvature and the quadratic torsion-provide the sufficient tools for understanding the contents of the classical solutions.

\section{COUPLED GRAVITATIONAL AND ELECTROMAGNETIC FIELDS}

As we discovered above, the material sources with spin may lead to certain inconsistencies in the framework of teleparallelism. Correspondingly, in order to be on the safe side, we will limit ourselves to the case of the spinless matter when the teleparallel gravity appears to be totally applicable. Of all the possible spinless matter, the Maxwell field clearly represents a very interesting and physically important case.

Accordingly, in our study of the spherically symmetric solutions, we will investigate the case when matter is represented by the electromagnetic field. The spin current of the electromagnetic field is trivial, whereas its energy momentum reads

$$
\left.\left.\Sigma_{\alpha}=\frac{\lambda}{2}\left[\left(e_{\alpha}\right\rfloor F\right) \wedge * F-\left(e_{\alpha}\right\rfloor * F\right) \wedge F\right] .
$$

Here, the electromagnetic vacuum constant (the "vacuum impedance")

$$
\lambda=\sqrt{\frac{\varepsilon_{0}}{\mu_{0}}},
$$

is defined in terms of the electric $\varepsilon_{0}$ and magnetic $\mu_{0}$ constants of the vacuum.

Using the standard spherically symmetric ansatz for the electromagnetic potential 1-form,

$$
\mathcal{A}=\frac{f}{A} \vartheta^{\hat{0}}=f d t
$$

with $f=f(r)$, we have for the field strength

$$
F=d \mathcal{A}=-\frac{f^{\prime}}{A B} \vartheta^{\hat{0}} \wedge \vartheta^{\hat{1}}, \quad * F=\frac{f^{\prime}}{A B} \vartheta^{\hat{2}} \wedge \vartheta^{\hat{3}}
$$

Then, we derive the Maxwell equation:

$$
d^{*} F=\frac{1}{r^{2} B^{3}} \frac{d}{d r}\left\{\frac{r^{2} B}{A} f^{\prime}\right\} \vartheta^{\hat{1}} \wedge \vartheta^{\hat{2}} \wedge \vartheta^{\hat{3}}=0 .
$$

On the other hand, a direct computation yields for the left-hand side of the teleparallel gravitational field equations

$$
\begin{aligned}
& D H_{\hat{0}}-E_{\hat{0}}=\frac{\left(U_{0}+U_{1}-2 U_{2}\right)}{6 \kappa B^{2}} \vartheta^{\hat{1}} \wedge \vartheta^{\hat{2}} \wedge \vartheta^{\hat{3}}, \\
& D H_{\hat{1}}-E_{\hat{1}}=\frac{U_{1}}{6 \kappa B^{2}} \vartheta^{\hat{0}} \wedge \vartheta^{\hat{2}} \wedge \vartheta^{\hat{3}}, \\
& D H_{\hat{2}}-E_{\hat{2}}=\frac{\left(U_{2}-U_{1}\right)}{6 \kappa B^{2}} \vartheta^{\hat{0}} \wedge \vartheta^{\hat{3}} \wedge \vartheta^{\hat{1}}, \\
& D H_{\hat{3}}-E_{\hat{3}}=\frac{\left(U_{2}-U_{1}\right)}{6 \kappa B^{2}} \vartheta^{\hat{0}} \wedge \vartheta^{\hat{1}} \wedge \vartheta^{\hat{2}} .
\end{aligned}
$$

Here, we have denoted

$$
\begin{aligned}
U_{0}:= & \frac{2}{A B r^{2}} \frac{d}{d r}\left\{A B r^{2}\left[\left(4 a_{1}-a_{2}\right) \frac{A^{\prime}}{A}-2\left(2 a_{1}+a_{2}\right) \frac{B^{\prime}}{B}\right]\right\}, \\
U_{1}:= & \frac{4}{r}\left(\left(a_{1}-a_{2}\right) \frac{A^{\prime}}{A}-\left(a_{1}+2 a_{2}\right) \frac{B^{\prime}}{B}\right)-\left(2 a_{1}+a_{2}\right)\left(\frac{A^{\prime}}{A}\right)^{2} \\
& -2\left(a_{1}+2 a_{2}\right)\left(\frac{B^{\prime}}{B}\right)^{2}+4\left(a_{1}-a_{2}\right) \frac{A^{\prime}}{A} \frac{B^{\prime}}{B}, \\
U_{2}:= & \frac{2}{A B r^{3}} \frac{d}{d r}\left\{A B r^{3}\left[\left(a_{1}-a_{2}\right) \frac{A^{\prime}}{A}-\left(a_{1}+2 a_{2}\right) \frac{B^{\prime}}{B}\right]\right\} .
\end{aligned}
$$

Substituting Eq. (8.4) into Eq. (8.1), we get explicitly the components of the electromagnetic energy-momentum current 3-form:

$$
\begin{aligned}
& \Sigma_{\hat{0}}=-\frac{\lambda}{2}\left(\frac{f^{\prime}}{A B}\right)^{2} \vartheta^{\hat{1}} \wedge \vartheta^{\hat{2}} \wedge \vartheta^{\hat{3}}, \\
& \Sigma_{\hat{1}}=\frac{\lambda}{2}\left(\frac{f^{\prime}}{A B}\right)^{2} \vartheta^{\hat{0}} \wedge \vartheta^{\hat{2}} \wedge \vartheta^{\hat{3}}, \\
& \Sigma_{\hat{2}}=-\frac{\lambda}{2}\left(\frac{f^{\prime}}{A B}\right)^{2} \vartheta^{\hat{0}} \wedge \vartheta^{\hat{3}} \wedge \vartheta^{\hat{1}}, \\
& \Sigma_{\hat{3}}=-\frac{\lambda}{2}\left(\frac{f^{\prime}}{A B}\right)^{2} \vartheta^{\hat{0}} \wedge \vartheta^{\hat{1}} \wedge \vartheta^{\hat{2}} .
\end{aligned}
$$


In the presence of a nontrivial electromagnetic field, we need, in addition to the above geometric invariants, an invariant description of the matter source configuration. As it is well known, there are two invariants of the Maxwell field. For the spherical ansatz (8.4), one of the invariants is trivial, $F \wedge F \equiv 0$, whereas the other reads

$$
F \wedge * F=-\left(\frac{f^{\prime}}{A B}\right)^{2} \eta
$$

\section{ANALYZING THE FIELD EQUATIONS}

The Maxwell equation (8.5) can be straightforwardly integrated. This yields

$$
f^{\prime}=\frac{q A}{r^{2} B}
$$

with $q$ an integration constant. Its value is determined by the total electric charge $Q$ of the source which is calculated as usual from the integral over the 2-sphere around the source:

$$
\begin{aligned}
Q & =\int_{S_{2}} \lambda * F=\int_{S_{2}} \lambda \frac{f^{\prime}}{A B} \vartheta^{\hat{2}} \wedge \vartheta^{\hat{3}}=\int_{S_{2}} \lambda q \sin \theta d \theta \wedge d \phi \\
& =4 \pi \lambda q .
\end{aligned}
$$

Inserting Eq. (9.1) into Eq. (8.17), we find for the Maxwell invariant

$$
F \wedge * F=-\frac{q^{2}}{r^{4} B^{4}} \eta
$$

From Eqs. (8.6)-(8.9) and Eqs. (8.13)-(8.16) we find, after making use of Eq. (9.1), and of some simple rearrangements:

$$
U_{0}=-\frac{6 \kappa \lambda q^{2}}{r^{4} B^{2}}, \quad U_{1}=\frac{3 \kappa \lambda q^{2}}{r^{4} B^{2}}, \quad U_{2}=0 .
$$

Using Eq. (8.10) in Eq. (9.4), we obtain the equation

$$
\begin{aligned}
& \frac{d}{d r}\left\{A B r^{2}\left[\left(4 a_{1}-a_{2}\right) \frac{A^{\prime}}{A}-2\left(2 a_{1}+a_{2}\right) \frac{B^{\prime}}{B}\right]\right\}=\frac{-3 \kappa \lambda q^{2} A}{r^{2} B} \\
& \quad=-3 \kappa \lambda q f^{\prime}
\end{aligned}
$$

where we have used Eq. (9.1) in the last step. Consequently, the first integral is straightforwardly obtained:

$$
\left(4 a_{1}-a_{2}\right) \frac{A^{\prime}}{A}-2\left(2 a_{1}+a_{2}\right) \frac{B^{\prime}}{B}=\frac{k_{1}-3 \kappa \lambda q f}{r^{2} A B},
$$

with $k_{1}$ an integration constant. Analogously, from Eqs. (8.12) and (9.4) we find another first integral:

$$
\left(a_{1}-a_{2}\right) \frac{A^{\prime}}{A}-\left(a_{1}+2 a_{2}\right) \frac{B^{\prime}}{B}=\frac{k_{2}}{r^{3} A B},
$$

with $k_{2}$ a second integration constant.

Let us introduce a new variable by

$$
\varphi:=k_{1}-3 \kappa \lambda q f \text {. }
$$

Differentiating this and using Eq. (9.1), we find the equation

$$
\varphi^{\prime}=-\frac{3 \kappa \lambda q^{2} A}{r^{2} B}
$$

Suppose $a_{1} a_{2} \neq 0$ (the special case $a_{1} a_{2}=0$ will be considered separately). Then, combining Eqs. (9.6) and (9.7), we finally get the system of first order equations

$$
\frac{A^{\prime}}{A}=\frac{1}{9 a_{1} a_{2} r^{2} A B}\left[\left(a_{1}+2 a_{2}\right) \varphi-2\left(2 a_{1}+a_{2}\right) \frac{k_{2}}{r}\right],
$$

$$
\frac{B^{\prime}}{B}=\frac{1}{9 a_{1} a_{2} r^{2} A B}\left[\left(a_{1}-a_{2}\right) \varphi-\left(4 a_{1}-a_{2}\right) \frac{k_{2}}{r}\right] .
$$

Together with Eq. (9.9), these equations comprise a system of three first order ordinary differential equations for the three unknown functions $A, B, f$. As an immediate consequence, the sum of Eqs. (9.10) and (9.11) yields

$$
(A B)^{\prime}=\frac{1}{9 a_{1} a_{2} r^{2}}\left[\left(2 a_{1}+a_{2}\right) \varphi-\left(8 a_{1}+a_{2}\right) \frac{k_{2}}{r}\right] .
$$

For the sake of completeness, we should recall that there is one more equation, which is derived by using Eq. (8.11) in Eq. (9.4):

$$
\begin{aligned}
\frac{3 \kappa \lambda q^{2}}{r^{4} B^{2}}= & \frac{4}{r}\left(\left(a_{1}-a_{2}\right) \frac{A^{\prime}}{A}-\left(a_{1}+2 a_{2}\right) \frac{B^{\prime}}{B}\right)-\left(2 a_{1}+a_{2}\right) \\
& \times\left(\frac{A^{\prime}}{A}\right)^{2}-2\left(a_{1}+2 a_{2}\right)\left(\frac{B^{\prime}}{B}\right)^{2}+4\left(a_{1}-a_{2}\right) \frac{A^{\prime}}{A} \frac{B^{\prime}}{B} .
\end{aligned}
$$

However, this last equation is satisfied automatically for the solutions (9.9)-(9.11) of the system. The easiest way to see this is to substitute Eqs. (9.10),(9.11) into Eq. (9.13). We then find

$$
\begin{gathered}
\left(a_{1}+2 a_{2}\right) \varphi^{2}-4\left(2 a_{1}+a_{2}\right) \frac{k_{2}}{r} \varphi+2\left(8 a_{1}+a_{2}\right) \frac{k_{2}^{2}}{r^{2}} \\
-9 a_{1} a_{2}\left(4 k_{2} A B-3 \kappa \lambda q^{2} A^{2}\right)=0 .
\end{gathered}
$$

Now, if we differentiate this equation, the result is identically zero by virtue of Eqs. (9.9), (9.12) and (9.10). However, since not only the equation itself vanishes for the solutions, but also its derivative, we still have to keep this equation. Ultimately it will turn out that Eq. (9.14) imposes certain relation between the various integration constants. 


\section{CONFORMALLY FLAT SOLUTIONS}

Let us study a very special case, when the metric functions are proportional. Namely,

$$
A=a_{0} B
$$

with a constant coefficient $a_{0}$. Then, the metric (7.1) describes the conformally flat spacetime geometry. The pair of equations (9.6) and (9.7) reduce to the system

$$
\begin{gathered}
3 a_{2} \frac{B^{\prime}}{B}=-\frac{\varphi}{a_{0} r^{2} B^{2}}, \\
3 a_{2} \frac{B^{\prime}}{B}=-\frac{k_{2}}{a_{0} r^{3} B^{2}} .
\end{gathered}
$$

Notice that the Lagrangian with the vanishing coupling constant $a_{2}=0$ belongs to the class of conformally covariant teleparallel gravity models (see Sec. VI). As a result, the overall factor of the tetrad, and hence of the metric, is undetermined. This is manifested in our spherically symmetric case as well: If we put $a_{2}=0$ in Eqs. (10.2),(10.3), we find a vanishing electromagnetic field $\varphi=0$, whereas the conformal metric factor $B$ remains completely arbitrary.

We will assume that the teleparallel model is not conformally covariant. Then $a_{2} \neq 0$, and the system (10.2),(10.3) yields the explicit electromagnetic function

$$
\varphi=\frac{k_{2}}{r} \text {. }
$$

Substituting this, together with Eq. (10.1), into Eq. (9.9), we get

$$
a_{0}=\frac{k_{2}}{3 \kappa \lambda q^{2}}
$$

Furthermore, the integration of Eq. (10.3) yields

$$
B^{2}=\frac{\kappa \lambda q^{2}}{a_{2} r^{2}}+k_{3}
$$

The new integration constant is fixed to be equal to zero $k_{3}$ $=0$, which follows from Eq. (9.13) after all the above is substituted. Thus, we obtain finally the conformally flat solution

$$
A^{2}=\frac{k_{2}^{2}}{9 \kappa \lambda q^{2} a_{2} r^{2}}, \quad B^{2}=\frac{\kappa \lambda q^{2}}{a_{2} r^{2}} .
$$

In order to understand this spacetime geometry, we have to compute the corresponding Riemannian curvature (recall that the total Riemann-Cartan curvature is identically zero). A direct calculation yields

$$
\widetilde{R}^{\hat{0} \hat{1}}=\frac{a_{2}}{\kappa \lambda q^{2}} \vartheta^{\hat{0}} \wedge \vartheta^{\hat{1}}, \quad \widetilde{R}^{\hat{2} \hat{3}}=-\frac{a_{2}}{\kappa \lambda q^{2}} \vartheta^{\hat{2}} \wedge \vartheta^{\hat{3}},
$$

with all other components of the curvature 2-form trivial. As we see, the curvature is everywhere regular, and the Riemann tensor has the double-duality property,

$$
\widetilde{R}_{\alpha \beta}{ }^{\gamma \delta}=\frac{1}{4} \eta^{\gamma \delta \rho \sigma} \eta_{\alpha \beta \mu \nu} \widetilde{R}_{\rho \sigma}^{\mu \nu} .
$$

The Riemannian Weyl tensor vanishes identically, as it is clearly seen from Eq. (7.8). This is consistent with the fact that the metric is conformally flat. Geometrically, the resulting spacetime is the direct product of the two 2-dimensional spaces of constant curvature, a hyperbolic space and a sphere. A solution of that type was originally described by Bertotti and Robinson [10,11] in the framework of general relativity theory.

In the teleparallel gravity, we have torsion as the basic field variable. It is straightforward to see that in the generalized Bertotti-Robinson solution (10.7), torsion is also constant with the quadratic invariant (7.11) given by

$$
T_{\alpha} \wedge * T^{\alpha}=-\frac{a_{2}}{\kappa \lambda q^{2}} \eta
$$

Note that the constant magnitude of the torsion is again described by the same combination of the coupling constants which determine the constant Riemannian curvature (10.8) of that solution.

The electromagnetic invariant (9.3), when using Eq. (10.7), demonstrates that the source is likewise represented by the "constant" electromagnetic field configuration:

$$
F \wedge * F=-\left(\frac{a_{2}}{\kappa \lambda q}\right)^{2} \eta
$$

\section{Vanishing coupling constant $a_{1}=0$}

Before we proceed to consider the general case, we have to analyze the special case when $a_{1} a_{2}=0$; see Sec. IX. When $a_{2}=0$, we have the conformally covariant theory, and consequently, the conformal factor of the metric remains undetermined. We will not consider such models which lack physical predictability.

When, however, $a_{1}=0$, the pair of equations (9.6) and (9.7) reduce to the system

$$
\begin{aligned}
& \frac{A^{\prime}}{A}+2 \frac{B^{\prime}}{B}=-\frac{\varphi}{a_{2} r^{2} A B}, \\
& \frac{A^{\prime}}{A}+2 \frac{B^{\prime}}{B}=-\frac{k_{2}}{a_{2} r^{3} A B} .
\end{aligned}
$$

This system yields the explicit electromagnetic function

$$
\varphi=\frac{k_{2}}{r}
$$

Substituting this into Eq. (9.9), we find the proportionality of the metric functions: 


$$
B=\frac{3 \kappa \lambda q^{2}}{k_{2}} A .
$$

This brings us then back to the results of the previous subsection.

\section{UNCHARGED SOLUTIONS}

From now on, we will confine our attention to the generic teleparallel models with $a_{1} a_{2} \neq 0$. Let us first obtain the configurations with zero charge, $q=0$. Then, from Eq. (9.8) we have $\varphi=k_{1}$, and Eq. (9.12) can be immediately integrated:

$$
A B=\frac{1}{9 a_{1} a_{2} r}\left[-\left(2 a_{1}+a_{2}\right) k_{1}+\left(8 a_{1}+a_{2}\right) \frac{k_{2}}{2 r}\right]+k_{3} .
$$

When we substitute this into Eq. (9.14), we find the value of the new integration constant explicitly:

$$
k_{3}=\frac{\left(a_{1}+2 a_{2}\right) k_{1}^{2}}{36 a_{1} a_{2} k_{2}} .
$$

\section{A. Special case: $8 a_{1}+a_{2}=0$}

Suppose the coupling constants satisfy

$$
8 a_{1}+a_{2}=0 .
$$

Then, Eqs. (11.1) and (11.2) yield

$$
A B=\frac{k_{1}}{12 a_{1}}\left(\frac{5 k_{1}}{8 k_{2}}-\frac{1}{r}\right) .
$$

Using this in Eq. (9.10), we derive the ordinary differential equation:

$$
\frac{A^{\prime}}{A}=\frac{2 k_{2}}{k_{1} r^{2}}\left(1-\frac{\frac{5 k_{1}}{8 k_{2}}}{\frac{1}{r}-\frac{5 k_{1}}{8 k_{2}}}\right) .
$$

The integration is straightforward, and the final solution for the metric function can be written in the form

$$
\begin{aligned}
& A^{2}=A_{0}^{2} e^{-5 r_{0} / 2 r}\left(\frac{r_{0}}{r}-1\right)^{5 / 2}, \\
& B^{2}=B_{0}^{2} e^{5 r_{0} / 2 r}\left(\frac{r_{0}}{r}-1\right)^{-1 / 2} .
\end{aligned}
$$

Here, $A_{0}, B_{0}$ and $r_{0}$ are arbitrary constants. For the sake of completeness, let us give their relation to the original (coupling and integration) constants:

$$
r_{0}=8 k_{2} / 5 k_{1}, \quad A_{0} B_{0}=5 k_{1}^{2} / 96 k_{2} a_{1} .
$$

This spacetime is not a black hole. The curvature tensor is singular at $r=0$ and at $r=r_{0}$, so although the metric component $g_{00}=-A^{2}$ vanishes at the finite radius $r=r_{0}$, this is not a horizon. Indeed, substituting $A$ and $B$ into Eq. (7.8), we get the Riemannian Weyl curvature:

$$
W=-\frac{r_{0}\left(48 r^{3}-136 r^{2} r_{0}+110 r r_{0}^{2}-25 r_{0}^{3}\right)}{4 r^{6} B_{0}^{2} e^{5 r_{0} / 2 r}\left(r_{0} / r-1\right)^{3 / 2}} .
$$

It is easy to see that it diverges at $r=r_{0}$. Analogously, for the torsion invariant (7.11), we find

$$
\begin{aligned}
T_{\alpha} \wedge * T^{\alpha} \\
\quad=\frac{r_{0}^{2}\left(172 r^{4}-564 r^{3} r_{0}+687 r^{2} r_{0}^{2}-370 r r_{0}^{3}+75 r_{0}^{4}\right)}{16 r^{8} B_{0}^{2} e^{5 r_{0} / 2 r}\left(r_{0} / r-1\right)^{7 / 2}} \eta .
\end{aligned}
$$

Thus, from both Riemannian and teleparallel viewpoints, the resulting geometry is singular at $r=0$ and at $r=r_{0}$.

\section{B. General case: $8 a_{1}+a_{2} \neq 0$}

When $8 a_{1}+a_{2} \neq 0$, we can use Eq. (11.2) to rewrite Eq. (11.1) as

$$
A B=\frac{k_{1}^{2}\left(8 a_{1}+a_{2}\right)}{18 a_{1} a_{2} k_{2}}\left(\frac{k_{2}}{k_{1} r}-\frac{\alpha}{2}\right)\left(\frac{k_{2}}{k_{1} r}-\frac{\beta}{2}\right),
$$

where we have introduced the constant parameters

$$
\begin{aligned}
& \alpha=\frac{2\left(2 a_{1}+a_{2}\right)+\sqrt{-18 a_{1} a_{2}}}{8 a_{1}+a_{2}}, \\
& \beta=\frac{2\left(2 a_{1}+a_{2}\right)-\sqrt{-18 a_{1} a_{2}}}{8 a_{1}+a_{2}} .
\end{aligned}
$$

In particular, we can easily see that

$$
\frac{\alpha+\beta}{2}=\frac{2\left(2 a_{1}+a_{2}\right)}{8 a_{1}+a_{2}}, \quad \frac{\alpha \beta}{4}=\frac{a_{1}+2 a_{2}}{2\left(8 a_{1}+a_{2}\right)} .
$$

From Eqs. (11.11),(11.12) we conclude that the product $a_{1} a_{2}$ must be negative. Substituting Eq. (11.10) into Eq. (9.10), we find the equation

$$
\frac{A^{\prime}}{A}=-\frac{k_{2}}{k_{1} r^{2}}\left[\frac{\alpha}{\frac{k_{2}}{k_{1} r}-\frac{\alpha}{2}}+\frac{\beta}{\frac{k_{2}}{k_{1} r}-\frac{\beta}{2}}\right] .
$$

Integration yields

$$
A=A_{0}\left(\frac{k_{2}}{k_{1} r}-\frac{\alpha}{2}\right)^{\alpha}\left(\frac{k_{2}}{k_{1} r}-\frac{\beta}{2}\right)^{\beta} .
$$

Using then Eq. (11.10), we find

$$
B=B_{0}\left(\frac{k_{2}}{k_{1} r}-\frac{\alpha}{2}\right)^{1-\alpha}\left(\frac{k_{2}}{k_{1} r}-\frac{\beta}{2}\right)^{1-\beta} .
$$


The integration constants are originally related by $A_{0} B_{0}$ $=k_{1}^{2}\left(8 a_{1}+a_{2}\right) / 18 a_{1} a_{2} k_{1}$. However, taking into account the possibility of scaling both the time and the radial coordinate by two arbitrary factors, the constants $A_{0}$ and $B_{0}$ can have any real value. In order to simplify the notation, it will be convenient to introduce $m=k_{2} / k_{1}$.

Performing a differentiation in Eq. (7.8), we find the nontrivial Riemannian Weyl curvature:

$$
W=\frac{m w}{r^{6} B_{0}^{2}}\left(\frac{m}{r}-\frac{\alpha}{2}\right)^{2 \alpha-4}\left(\frac{m}{r}-\frac{\beta}{2}\right)^{2 \beta-4},
$$

where we denoted the polynomial

$$
\begin{aligned}
w:= & \frac{1}{8}\left\{32 m^{3}(\alpha+\beta)(\alpha+\beta-1)+4 m^{2} r[(\alpha+\beta)(3-2(\alpha\right. \\
& +\beta)-16 \alpha \beta)+12 \alpha \beta]+2 m r^{2}[8 \alpha \beta(2 \alpha \beta+\alpha+\beta-1) \\
& \left.\left.-(\alpha+\beta)^{2}\right]+3 r^{3} \alpha \beta(\alpha+\beta-4 \alpha \beta)\right\} \\
= & \frac{-3 a_{2}}{\left(8 a_{1}+a_{2}\right)^{2}}\left[a_{1}(r-4 m)\left(3 r^{2}-8 m r+8 m^{2}\right)+2 a_{2}(r\right. \\
& \left.-m)^{2}(3 r-8 m)\right] .
\end{aligned}
$$

Analogously, for the quadratic torsion invariant (7.11), we find

$$
T_{\alpha} \wedge * T^{\alpha}=\frac{m^{2} \mathcal{T}}{r^{6} B_{0}^{2}}\left(\frac{m}{r}-\frac{\alpha}{2}\right)^{2 \alpha-4}\left(\frac{m}{r}-\frac{\beta}{2}\right)^{2 \beta-4} \eta,
$$

where we have another polynomial defined as

$$
\begin{aligned}
\mathcal{T}:= & m^{2}\left[3(\alpha+\beta)^{2}+8(1-\alpha-\beta)\right]+2 m r\left[(\alpha+\beta)^{2}-(\alpha+\beta)\right. \\
& \times(2+3 \alpha \beta)+4 \alpha \beta]+r^{2}\left[(\alpha+\beta)^{2} / 2+\alpha \beta(3 \alpha \beta-2(\alpha\right. \\
& +\beta))]=\frac{12}{\left(8 a_{1}+a_{2}\right)^{2}}\left[a_{1}^{2}(r-4 m)^{2}+2 a_{2}^{2}(r-m)^{2}\right] .
\end{aligned}
$$

\section{COUPLING CONSTANTS, INVARIANTS, AND SINGULARITIES}

The values of the constants $\alpha$ and $\beta$ are crucial for understanding the spacetime geometry of the solutions obtained above, since they determine the behavior of the metric functions (11.15) and (11.16). A straightforward analysis of Eqs. (11.11),(11.12) shows that we can simplify those formulas to the following equivalent ones:

$$
\begin{aligned}
& \alpha=\frac{2\left(a_{1}+\sqrt{-2 a_{1} a_{2}}\right)}{4 a_{1}+\sqrt{-2 a_{1} a_{2}}}, \\
& \beta=\frac{2\left(a_{1}-\sqrt{-2 a_{1} a_{2}}\right)}{4 a_{1}-\sqrt{-2 a_{1} a_{2}}} .
\end{aligned}
$$

Moreover, one can eliminate $\sqrt{-2 a_{1} a_{2}}$ from Eqs. (12.1),(12.2), and express one constant directly in terms of the other:

$$
\alpha=\frac{4-5 \beta}{5-4 \beta}, \quad \text { or equivalently, } \quad \beta=\frac{4-5 \alpha}{5-4 \alpha} .
$$

From these simple derivations, we can immediately establish a number of important consequences. Since $a_{1} a_{2}<0$ (in order to have real solutions), we have to analyze the two cases for the original coupling constants: (a) $a_{1}>0, a_{2}<0$, and (b) $a_{1}<0, a_{2}>0$.

For $a_{1}>0, a_{2}<0$, we see from Eq. (12.1) that $\alpha$ is positive. Moreover,

$$
\frac{1}{2}<\alpha<2 \text { for all } a_{1}, a_{2} .
$$

At the same time, Eq. (12.2) shows that

$$
\begin{aligned}
0<\beta<\frac{1}{2} & \text { for } \quad a_{1}>-2 a_{2}, \\
\beta>2 & \text { for } \quad a_{1}<-a_{2} / 8, \\
\beta<0 & \text { for } \quad-a_{2} / 8<a_{1}<-2 a_{2} .
\end{aligned}
$$

For $a_{1}=-2 a_{2}$, we see that $\beta=0$, whereas when $a_{1}=$ $-a_{2} / 8$, we return to the exceptional case considered in Sec. XI A (then, $\beta$ formally diverges).

For $a_{1}<0, a_{2}>0$, we analogously find from Eq. (12.2) that now $\beta$ is positive,

$$
\frac{1}{2}<\beta<2 \text { for all } a_{1}, a_{2},
$$

whereas Eq. (12.1) yields

$$
\begin{aligned}
0<\alpha<\frac{1}{2} & \text { for } \quad a_{1}<-2 a_{2}, \\
\alpha>2 & \text { for } \quad a_{1}>-a_{2} / 8, \\
\alpha<0 & \text { for } \quad-2 a_{2}<a_{1}<-a_{2} / 8 .
\end{aligned}
$$

For $a_{1}=-2 a_{2}$, we see that $\alpha=0$, whereas when $a_{1}=$ $-a_{2} / 8$, we again obtain the exceptional case considered in Sec. XI A (then $\alpha$ becomes infinite).

It is important to find the zeros of the metric coefficient $g_{00}=-A^{2}$, since these values determine the position of a possible horizon. For the solutions under consideration, $A$ always has one or two zeros: (a) at $r=2 m / \alpha$ when $\alpha>0$, and/or (b) at $r=2 m / \beta$ if $\beta>0$. The function (11.15) would not have zeros only in the case when both $\alpha \leqslant 0$ and $\beta \leqslant 0$, but this never happens according to the above analysis.

In order to decide whether a zero of $g_{00}$ corresponds to a horizon, we have to study the behavior of the curvature and torsion invariants at that value of the radial coordinate. As it 
is clear from Eq. (11.17), the Riemannian curvature diverges at the zeros of $A$, unless the polynomial (11.18) also vanishes there. Hence, it is important to find the value of the polynomial (11.18) at the zeros of the function $A$. A direct substitution yields

$$
\begin{aligned}
& w(2 m / \alpha)=2 m^{3}(\alpha-\beta)^{2} \frac{2 \alpha^{2}-3 \alpha+1}{\alpha^{2}}, \\
& w(2 m / \beta)=2 m^{3}(\alpha-\beta)^{2} \frac{2 \beta^{2}-3 \beta+1}{\beta^{2}} .
\end{aligned}
$$

Since $\alpha$ can never be equal to $\beta$ (for any nonzero coupling constants $a_{1}, a_{2}$ ), we conclude from Eq. (12.12) that the polynomial $w$ can have common zeros with $A$ only when either $\alpha$ or $\beta$ is equal to 1 or $1 / 2$. Equations (12.1),(12.2) show that this is possible only when $2 a_{1}+a_{2}=0$ (then either $\alpha$ or $\beta$ is equal to 1 ), or when one of the two constants $a_{1}$ or $a_{2}$ vanishes.

Analogously, for the torsion quadratic invariant we find

$$
\begin{aligned}
& \mathcal{T}(2 m / \alpha)=m^{2}(\alpha-\beta)^{2} \frac{3 \alpha^{2}-4 \alpha+2}{\alpha^{2}}, \\
& \mathcal{T}(2 m / \beta)=m^{2}(\alpha-\beta)^{2} \frac{3 \beta^{2}-4 \beta+2}{\beta^{2}} .
\end{aligned}
$$

As one can easily see, these quantities are nonvanishing for any choice of the constants $\alpha, \beta$. Correspondingly, the torsion invariant is always singular at the zeros of $g_{00}$ in all teleparallel gravity models.

\section{A. General relativity limit: Schwarzschild black hole}

As we have mentioned in Sec. V, the specific choice (5.1) of the coupling constants gives rise to a teleparallel model which is called the teleparallel equivalent of GR. Accordingly, when $a_{1}=-1$ and $a_{2}=2$, we find from Eqs. (11.11),(11.12) that

$$
\alpha=-1, \quad \beta=1 .
$$

Consequently, the metric functions reduce to

$$
A=A_{0}\left(\frac{m}{r}-\frac{1}{2}\right)\left(\frac{m}{r}+\frac{1}{2}\right)^{-1}, \quad B=B_{0}\left(\frac{m}{r}+\frac{1}{2}\right)^{2} .
$$

Furthermore, the polynomial (11.18) now reads

$$
w=-\frac{3 r}{2}(2 m-r)^{2}=-6 r^{3}\left(\frac{m}{r}-\frac{1}{2}\right)^{2},
$$

and consequently, the Riemannian Weyl curvature is described by

$$
W=-\frac{6 m r^{3}}{B_{0}^{2}(m+r / 2)^{6}} .
$$

The most important observation is that the Riemannian curvature is thus regular at the zero $r=2 \mathrm{~m}$ of the metric function $A(r)$, which means that we have a horizon here. The resulting geometry then describes the well known Schwarzschild black hole with the horizon at $r=2 \mathrm{~m}$. The singularity at $r=-2 m$ is the usual point-mass source singularity at the origin.

Since we are dealing with teleparallel gravity, it is necessary also to analyze the behavior of torsion. A direct substitution of Eq. (12.14) into Eqs. (11.19),(11.20) yields

$$
T_{\alpha} \wedge * T^{\alpha}=\frac{m^{2}\left(3 r^{2}-8 m r+8 m^{2}\right) r^{2}}{B_{0}^{2}(m+r / 2)^{6}(m-r / 2)^{2}} \eta .
$$

As we see, the torsion invariant diverges not only at the origin $r=-2 m$, but also at the Schwarzschild horizon $r$ $=2 \mathrm{~m}$. Unlike the "regularizing" effect of the polynomial (12.16) in the curvature invariant, the analogous polynomial in the numerator of Eq. (12.18) does not help to remove the singularity of the torsion invariant.

We emphasize this fact as the main difference between the standard general relativistic description of the Schwarzschild black hole and its teleparallel counterpart. The horizon is a regular surface from the viewpoint of the Riemannian geometry, but it is singular from the viewpoint of teleparallel gravity.

\section{B. No black holes in teleparallel gravity?}

Returning to the case of the general teleparallel Lagrangian, we may ask if a (class of) model exists with a specific choice of the coupling constants for which the above solutions describe a black hole. As we see from Eq. (11.17), the curvature is singular at both zeros of the metric function $A$, i.e., at $r=2 \mathrm{~m} / \alpha$ and at $r=2 \mathrm{~m} / \beta$, when

$$
\alpha<2 \text { and } \beta<2 \text {. }
$$

Correspondingly, recalling the results of the beginning of the current section, the teleparallel Lagrangians with $\left|a_{1}\right|$ $>\left|a_{2}\right| / 8$ do not have spherically symmetric solutions describing black holes.

There is, in principle, a possibility that the configurations with

$$
\alpha>2 \text { or } \beta>2
$$

may turn out to be black holes. Note that both $\alpha$ and $\beta$ cannot be simultaneously greater than 2 , as was shown above in this section. When these conditions are satisfied, the curvature becomes regular either at $r=2 \mathrm{~m} / \alpha$ or at $r=2 \mathrm{~m} / \beta$. However, the problem is that the corresponding surface $(r$ $=2 \mathrm{~m} / \alpha$ or $r=2 \mathrm{~m} / \beta$ ) would have an infinite area. This is different from what we usually expect from a horizon surface. 
Consequently, we come to the conclusion that for all possible values of the coupling constants $a_{1}$ and $a_{2}$-except for the special case $a_{1}=-1$ and $a_{2}=2$-the uncharged spherically symmetric solutions (11.6),(11.7), as well as Eqs. (11.15),(11.16) of the general teleparallel model (3.4), do not describe black hole configurations. In this sense, the socalled teleparallel equivalent of GR is distinguished among all other teleparallel models as the only theory which admits black holes.

\section{CHARGED SOLUTIONS: GENERAL RELATIVITY LIMIT}

Let us now return to the charged solutions determined by the system (9.9)-(9.11). In view of the above result, one can expect that the most physically interesting case, within the class of the general teleparallel theories (3.4), corresponds to the teleparallel equivalent of GR. Accordingly, we will confine our attention now to the case $a_{1}=-1, a_{2}=2$, or equivalently to Eq. (12.14). Then, Eqs. (9.11),(9.12) are reduced to

$$
\begin{aligned}
\frac{A^{\prime}}{A} & =-\frac{\varphi}{6 r^{2} A B}, \\
\frac{B^{\prime}}{B} & =\frac{1}{6 r^{2} A B}\left(\varphi-\frac{2 k_{2}}{r}\right), \\
(A B)^{\prime} & =-\frac{k_{2}}{3 r^{3}} .
\end{aligned}
$$

The last equation is easily integrated, yielding

$$
A B=\frac{1}{6}\left(\frac{k_{2}}{r^{2}}+k_{3}\right),
$$

where $k_{3}$ is a new integration constant. Combining Eqs. (9.9) and (13.1), we can eliminate $B$ to find

$$
A^{\prime} A=\frac{\varphi^{\prime} \varphi}{18 \kappa \lambda q^{2}} .
$$

This immediately yields the first integral

$$
A^{2}=\frac{\varphi^{2}+k_{4}}{18 \kappa \lambda q^{2}} .
$$

The new integration constant $k_{4}$ can have any sign, as well as be equal to zero. Each case will be studied separately below. Substituting Eqs. (13.6) and (13.4) into the additional equation (9.14), we find the following relation between the integration constants:

$$
4 k_{2} k_{3}=k_{4} .
$$

The final step is to find the function $\varphi(r)$ explicitly. Substituting Eqs. (13.6) and (13.4) into Eq. (9.9), and taking into account the condition (13.7), we obtain the differential equation

$$
\varphi^{\prime}=-\frac{k_{4}+\varphi^{2}}{k_{2}\left[1+k_{4}\left(r / 2 k_{2}\right)^{2}\right]},
$$

The form of the solution depends crucially on the value of $k_{4}$.

\section{A. Negative constant $k_{4}$}

For negative integration constant, $k_{4}=-\left|k_{4}\right|$, the solution of Eq. (13.8) reads

$$
\varphi(r)=\sqrt{\left|k_{4}\right|} \frac{\left[k_{5}\left(r+r_{0}\right)^{2}+\left(r-r_{0}\right)^{2}\right]}{\left[k_{5}\left(r+r_{0}\right)^{2}-\left(r-r_{0}\right)^{2}\right]},
$$

where $k_{5}$ is a new integration constant, and we denoted

$$
r_{0}:=\frac{2 k_{2}}{\sqrt{\left|k_{4}\right|}} .
$$

Substituting Eq. (13.9) into Eqs. (13.6) and (13.4), we find the metric functions:

$$
\begin{aligned}
& A^{2}=\frac{2 k_{5}\left|k_{4}\right|}{9 \kappa \lambda q^{2}}\left[\frac{r^{2}-r_{0}^{2}}{k_{5}\left(r+r_{0}\right)^{2}-\left(r-r_{0}\right)^{2}}\right]^{2}, \\
& B^{2}=\frac{\kappa \lambda q^{2}}{32 k_{5} r_{0}^{2} r^{4}}\left[k_{5}\left(r+r_{0}\right)^{2}-\left(r-r_{0}\right)^{2}\right]^{2} .
\end{aligned}
$$

In order to have a correct signature of the metric (7.1), we must assume that $k_{5}>0$.

As we see, the metric coefficient $g_{00}=-A^{2}$ has zeros at $r= \pm r_{0}$. These values can qualify for the positions of a horizon, and in order to clarify this we have to study the behavior of the geometric and electromagnetic invariants. The quadratic torsion invariant (7.11) is as follows:

$$
T_{\alpha} \wedge * T^{\alpha}=\frac{128 k_{5} r_{0}^{4} r^{2} \check{\mathcal{T}}}{\kappa \lambda q^{2}\left(r^{2}-r_{0}^{2}\right)^{2}\left[k_{5}\left(r+r_{0}\right)^{2}-\left(r-r_{0}\right)^{2}\right]^{4}} \eta,
$$

where the polynomial reads

$$
\begin{aligned}
\check{\mathcal{T}}= & k_{5}^{2}\left(3 r^{2}-4 r r_{0}+2 r_{0}^{2}\right)\left(r+r_{0}\right)^{4}+2 k_{5}\left(3 r^{2}-2 r_{0}^{2}\right)\left(r^{2}-r_{0}^{2}\right)^{2} \\
& +\left(3 r^{2}+4 r r_{0}+2 r_{0}^{2}\right)\left(r-r_{0}\right)^{4} .
\end{aligned}
$$

On the other hand, for the Maxwell invariant (9.3) we find

$$
F \wedge * F=-\frac{1024 k_{5}^{2} r_{0}^{4} r^{4}}{(\kappa \lambda q)^{2}\left[k_{5}\left(r+r_{0}\right)^{2}-\left(r-r_{0}\right)^{2}\right]^{4}} \eta .
$$

As far as the Riemannian curvature is concerned, everything becomes more transparent if we make the coordinate transformation defined by 


$$
\begin{aligned}
& \rho=\sqrt{\frac{\kappa \lambda q^{2}}{8 k_{5}}}\left[\frac{k_{5}\left(r+r_{0}\right)^{2}-\left(r-r_{0}\right)^{2}}{2 r_{0} r}\right], \\
& \bar{t}=-\frac{1}{3 c\left(k_{5}-1\right)} \sqrt{\frac{2 k_{5}\left|k_{4}\right|}{\kappa \lambda q^{2}} t .}
\end{aligned}
$$

Then, the line element transforms into the standard ReissnerNordström form:

$$
d s^{2}=-h^{2} c^{2} d \bar{t}^{2}+\frac{1}{h^{2}} d \rho^{2}+\rho^{2}\left(d \theta^{2}+\sin ^{2} \theta d \phi^{2}\right),
$$

with

$$
h^{2}=1-\frac{2 G m}{c^{2} \rho}+\frac{G Q^{2}}{4 \pi \varepsilon_{0} c^{4} \rho^{2}} .
$$

The mass parameter is here introduced by

$$
\frac{G m}{c^{2}}=\left(k_{5}+1\right) \sqrt{\frac{\kappa \lambda q^{2}}{8 k_{5}}},
$$

whereas the charge $Q$ of the source is given, as usual, by Eq. (9.2).

It is easy to see that the values $r= \pm r_{0}$ correspond to the two zeros of the metric function $h^{2}(\rho)$. As a result, we conclude that these values give the position of the horizon because both the Maxwell invariant (13.15) and the Riemannian curvature (as it is well known) are regular there. However, it is remarkable that the torsion (13.13) is again singular at those surfaces, just like in the uncharged solutions considered earlier.

\section{B. Positive constant $k_{4}$}

For a positive integration constant $k_{4}=\left|k_{4}\right|$, the integration of Eq. (13.8) is also straightforward, yielding

$$
\varphi(r)=\sqrt{\left|k_{4}\right| \frac{\left[k_{5}\left(r^{2}-r_{0}^{2}\right)+2 r r_{0}\right]}{\left[r^{2}-r_{0}^{2}-2 k_{5} r r_{0}\right]} .}
$$

Here, $k_{5}$ is a new integration constant (which is different from the constant $k_{5}$ introduced in the previous subsection), and we use the same $r_{0}$ as defined in Eq. (13.10). We then easily find the metric functions, using Eqs. (13.6) and (13.4):

$$
\begin{aligned}
& A^{2}=\frac{\left(1+k_{5}^{2}\right)\left|k_{4}\right|}{18 \kappa \lambda q^{2}}\left[\frac{r^{2}+r_{0}^{2}}{r^{2}-r_{0}^{2}-2 k_{5} r r_{0}}\right]^{2}, \\
& B^{2}=\frac{\kappa \lambda q^{2}}{8\left(1+k_{5}^{2}\right) r_{0}^{2} r^{4}}\left[r^{2}-r_{0}^{2}-2 k_{5} r r_{0}\right]^{2} .
\end{aligned}
$$

Now, we may notice that the coordinate transformation

$$
\begin{aligned}
& \rho=\sqrt{\frac{\kappa \lambda q^{2}}{2\left(1+k_{5}^{2}\right)}}\left[\frac{r^{2}-r_{0}^{2}-2 k_{5} r r_{0}}{2 r_{0} r}\right], \\
& \bar{t}=\frac{1}{3 c} \sqrt{\frac{\left(1+k_{5}^{2}\right)\left|k_{4}\right|}{2 \kappa \lambda q^{2}} t,}
\end{aligned}
$$

again brings the line element to the Reissner-Nordström form (13.18), where this time the mass is introduced via

$$
\frac{G m}{c^{2}}=-k_{5} \sqrt{\frac{\kappa \lambda q^{2}}{2\left(1+k_{5}^{2}\right)}} .
$$

This shows that $k_{5}$ should be negative in this case.

We will not give the curvature, torsion, and Maxwell invariants explicitly, but their qualitative behavior is the same as in the previous case: The torsion invariant is again singular on a Riemannian horizon of the corresponding ReissnerNordström black hole.

\section{Vanishing constant $\boldsymbol{k}_{\mathbf{4}}$}

For the sake of completeness, it remains to consider the case of vanishing integration constant $k_{4}=0$. Then, as follows from Eq. (13.7), one should also have either $k_{2}=0$ or $k_{3}=0$.

$$
\text { 1. Case: } k_{4}=0, k_{2}=0
$$

In this case, Eq. (13.8) is easily solved to give

$$
\varphi=\frac{k_{3} r}{k_{5} r-1},
$$

with an arbitrary integration constant $k_{5}$. Correspondingly, Eq. (13.6) and (13.4) yield

$$
\begin{aligned}
& A^{2}=\frac{k_{3}^{2} r^{2}}{18 \kappa \lambda q^{2}\left(k_{5} r-1\right)^{2}}, \\
& B^{2}=\frac{\kappa \lambda q^{2}\left(k_{5} r-1\right)^{2}}{2 r^{2}} .
\end{aligned}
$$

The coordinate transformation

$$
\begin{aligned}
& \rho=-\sqrt{\frac{\kappa \lambda q^{2}}{2}}\left(k_{5} r-1\right), \\
& \bar{t}=\frac{k_{3} t}{3 c k_{5} \sqrt{2 \kappa \lambda q^{2}}},
\end{aligned}
$$

brings the metric to the extremal Reissner-Nordström line element 


$$
\begin{aligned}
& d s^{2}=-\left(1-\frac{\sqrt{\frac{\kappa \lambda q^{2}}{2}}}{\rho}\right)^{2} d \bar{t}^{2}+\left(1-\frac{\sqrt{\frac{\kappa \lambda q^{2}}{2}}}{\rho}\right)^{-2} d \rho^{2} \\
&+\rho^{2}\left(d \theta^{2}+\sin ^{2} \theta d \phi^{2}\right) . \\
& \text { 2. Case }: \boldsymbol{k}_{4}=\mathbf{0}, \boldsymbol{k}_{3}=\mathbf{0}
\end{aligned}
$$

Analogously, Eq. (13.8) is integrated,

$$
\varphi=\frac{k_{2}}{r+k_{5}},
$$

with an integration constant $k_{5}$. Then, Eqs. (13.6) and (13.4) yield the metric functions:

$$
\begin{aligned}
A^{2} & =\frac{k_{2}^{2}}{18 \kappa \lambda q^{2}\left(r+k_{5}\right)^{2}}, \\
B^{2} & =\frac{\kappa \lambda q^{2}\left(r+k_{5}\right)^{2}}{2 r^{4}} .
\end{aligned}
$$

The coordinate transformation

$$
\begin{aligned}
& \rho=\sqrt{\frac{\kappa \lambda q^{2}}{2}\left(1+\frac{k_{5}}{r}\right),} \\
& \bar{t}=\frac{k_{2} t}{3 c k_{5} \sqrt{2 \kappa \lambda q^{2}}},
\end{aligned}
$$

again yields the extremal Reissner-Nordström metric (13.32).

In both cases, the torsion diverges at $\rho=\sqrt{\kappa \lambda q^{2} / 2}$, whereas this surface appears to be regular from the Riemannian point of view.

\section{DISCUSSION AND CONCLUSIONS}

In this paper we have studied the general teleparallel gravity model within the framework of the MAG theory. A similar analysis of "embedding" teleparallelism into the Poincaré gauge theory was performed in [6] within the framework of the Lagrangian formalism, and also by using the Hamiltonian methods in [20,21]. Generalizing the previous work, we consider the full 3-parameter teleparallel Lagrangian without a priori restricting the coupling constants $a_{1}, a_{2}, a_{3}$. The main motivation for this was to determine the place and significance of the so-called teleparallel GRequivalent model which is specialized by the values (5.1). It is well known that, for obvious reasons, the GR-equivalent teleparallel theory is satisfactorily supported by observations.

Our study reveals a qualitative feature which distinguishes the teleparallel GR equivalent among other models: The spherically symmetric solutions (charged and uncharged) describe black hole configurations only for the special choice (5.1) of the coupling constants. We have thus demonstrated that a generic teleparallel model does not admit black holes. There exists, though, a family of completely regular solutions which appears to be a direct generalization of the Bertotti-Robinson solution.

Another result obtained concerns the behavior of the curvature and torsion invariants in the general teleparallel model. We find that the quadratic torsion invariant displays a much worse singularity structure than one could expect from the analysis of the Riemannian curvature invariants. In particular, even in the teleparallel GR-equivalent model, the black hole solutions have torsion singularities on a horizon surface which is, however, regular from the point of view of the curvature. This striking result raises a question about the geometrical and physical meaning of the torsion singularities in teleparallel gravity.

Finally, it seems worthwhile to note that, although the teleparallel GR-equivalent model has a number of nice features which distinguishes it among the general teleparallel theories, it still has a consistency problem of coupling of matter with spin to the teleparallel gravitational field. As a matter of fact, one can argue that there is no such problem because, being a gauge theory of the group of translations, teleparallelism is thus, by definition, related only to the energy-momentum current. And indeed, teleparallelism turns out to be completely consistent for the case of spinless matter, which is characterized solely by the energy momentum. From this point of view, teleparallelism appears to be not applicable to matter sources with spin, and our analysis has clearly demonstrated that point.

Note added in proof. The spherically symmetric solutions in teleparallel gravity were also studied in Ref. [22] and Ref. [23]. Our results do not agree with the latter reference.

\section{ACKNOWLEDGMENTS}

The work of Y.N.O. was supported by FAPESP. J.G.P. thanks FAPESP and CNPq for partial financial support.
[1] K. Hayashi and T. Nakano, Prog. Theor. Phys. 38, 491 (1967).

[2] K. Hayashi and T. Shirafuji, Phys. Rev. D 19, 3524 (1979).

[3] V. C. de Andrade and J. G. Pereira, Phys. Rev. D 56, 4689 (1997).

[4] V. C. de Andrade, L. C. T. Guillen, and J. G. Pereira, Phys. Rev. Lett. 84, 4533 (2000).

[5] V. C. de Andrade, L. C. T. Guillen, and J. G. Pereira, Phys. Rev. D 64, 027502 (2001).

[6] W. Kopczyński, J. Phys. A 15, 493 (1982).
[7] J. M. Nester, Class. Quantum Grav. 5, 1003 (1988).

[8] F. W. Hehl, J. D. McCrea, E. W. Mielke, and Y. Ne'eman, Phys. Rep. 258, 1 (1995).

[9] Yu. N. Obukhov, E. J. Vlachynsky, W. Esser, and F. W. Hehl, Phys. Rev. D 56, 7769 (1997).

[10] B. Bertotti, Phys. Rev. 116, 1331 (1959).

[11] I. Robinson, Bull. Acad. Pol. Sci., Ser. Sci., Math., Astron. Phys. 7, 351 (1959).

[12] G. G. L. Nashed, Phys. Rev. D 66, 064015 (2002). 
[13] N. Toma, Prog. Theor. Phys. 86, 659 (1991).

[14] T. Kawai and N. Toma, Prog. Theor. Phys. 87, 583 (1992).

[15] T. Dereli and R. W. Tucker, Phys. Lett. 110B, 206 (1982).

[16] H. T. Nieh, Phys. Lett. 88A, 388 (1982).

[17] Yu. N. Obukhov, Phys. Lett. 90A, 13 (1982).

[18] I. L. Shapiro, Phys. Rep. 357, 113 (2001).

[19] J. G. Pereira, T. Vargas, and C. M. Zhang, Class. Quantum Grav. 18, 833 (2001).
[20] M. Blagojević and M. Vasilić, Phys. Rev. D 64, 044010 (2001).

[21] M. Blagojević and M. Vasilić, Class. Quantum Grav. 19, 3723 (2002).

[22] U. Muench, F. Gronwald, and F. W. Hehl, Gen. Relativ. Gravit. 30, 933 (1998).

[23] Y. Itin, Int. J. Mod. Phys. D 10, 547 (2001). 